\title{
Carbocyclization of Aromatic Iodides, Bicyclic Alkenes and Benzynes Involving a Palladium-Catalyzed C-H Bond Activation as a Key Step
}

Sivakolundu Bhuvaneswari, Masilamani Jeganmohan and Chien-Hong Cheng*

Department of Chemistry, National Tsing Hua University, Hsinchu 30013, Taiwan

\section{Supporting Information}

\section{Table of Contents}

S2 - S3 Experimental Section, Regio and Stereoselective Studies

S4 - S10 Spectral Data of Compounds 4a-r

S10 Procedure for Deoxyaromatization Reaction and Spectral Data of

Compounds $\mathbf{7 a}$ and $\mathbf{7 b}$

S11 Procedure for the Isolation of Intermediate $\mathbf{C}$

S12 - $31 \quad{ }^{1} \mathrm{H}$ and ${ }^{13} \mathrm{C}$ NMR Spectra of All Compounds 


\section{Experimental Section}

General Procedure for the Carbocyclization Reaction of Aromatic Iodides, Bicyclic Alkenes and Benzynes. A 25-mL round-bottomed side-arm flask containing $\mathrm{Pd}(\mathrm{dba})_{2} \quad(0.005 \mathrm{mmol}), \mathrm{P}(2-\mathrm{furyl})_{3}(0.020 \mathrm{mmol})$ and $\mathrm{CsF}(4.00 \mathrm{mmol})$ was evacuated and purged with nitrogen gas three times. To the flask were then added aromatic iodides (1) (1.00 mmol), bicyclic alkenes 2 (for 2a and 2c $1.50 \mathrm{mmol}, \mathbf{2 b}$ $1.00 \mathrm{mmol})$, benzyne precursor $3(1.10 \mathrm{mmol})$ and $\mathrm{CH}_{3} \mathrm{CN}(3.0 \mathrm{~mL})$ via syringes. The reaction mixture was allowed to stir at room temperature for $10 \mathrm{~h}$. At the end of the reaction, the reaction mixture was diluted with $\mathrm{CH}_{2} \mathrm{Cl}_{2}$, filtered through Celite and silica gel, and the filtrate was concentrated. The crude residue was purified through a silica gel column using hexanes and ethyl acetate as eluent to give pure 4 .

Spectral data and copy of ${ }^{1} \mathrm{H}$ and ${ }^{13} \mathrm{C}$ NMR spectra of all compounds $4 \mathbf{a}-\mathbf{r}$ are listed below (pages $4-31$ ). All of these products 4a-r decomposed during melting point determination, likely undergoing retro Diels-Alder reactions, at temperature $>100^{\circ} \mathrm{C}$.

\section{Regioselective Studies}

In this reaction, two different kinds of regioisomers are possible $\mathbf{4 a}$ or $\mathbf{4 a}$ ', but we observed only $\mathbf{4 a}$. The regiochemistry of compound $\mathbf{4 a}$ was established by ${ }^{1} \mathrm{H}$ NMR NOE experiments.

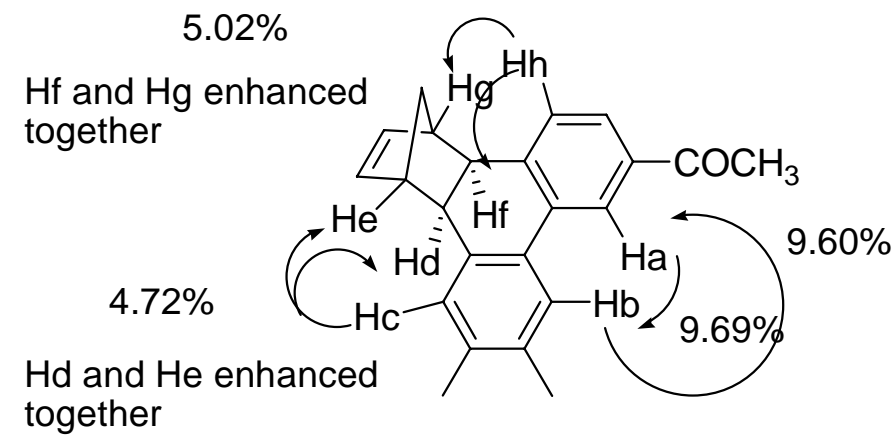




\section{Stereoselective Studies}

The catalytic reaction is highly stereoselective, giving the exo isomer as the exclusive product. Exo stereochemistry of this product was established on the basis of the coupling constants of protons $\mathrm{H}-1$ and $\mathrm{H}-2$ and $\mathrm{H}-1$ ' and $\mathrm{H}-2$ '. It is well-known that for norbornene derivatives the coupling constant between the endo proton at C-2' and the bridgehead proton at C-1' is nearly zero, whereas the value between the exo and a bridgehead proton is ca. $3 \mathrm{~Hz}$ and can be clearly observed. For compounds $\mathbf{4 a}$, $\mathbf{4 d}$ and $\mathbf{4 j}$, the $\mathrm{C} 1$ and $\mathrm{C} 1$ ' protons all appear as singlets in their ${ }^{1} \mathrm{H}$ NMR spectra at $\delta$ 3., thereby confirming the exo stereochemistry. ${ }^{1}$ A similar type of exo stereoisomers was observed for compounds $\mathbf{4 b}-\mathbf{c}$, $\mathbf{4 e -} \mathbf{i}$, and $\mathbf{4 k - \mathbf { r }}$.
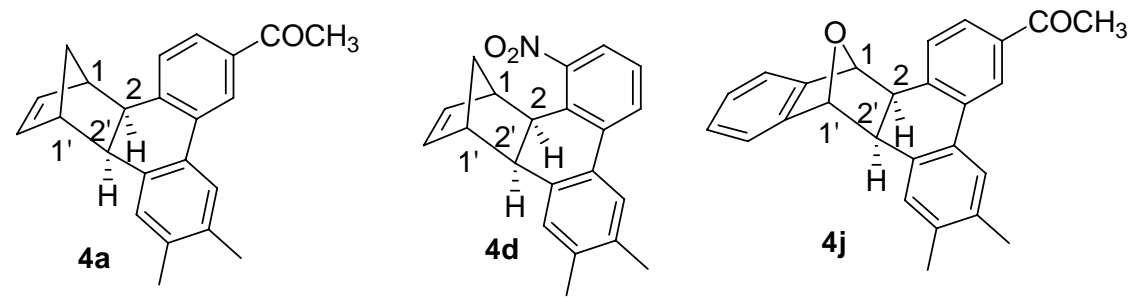

\section{References}

1. (a) Smith, C. D. J. Am. Chem. Soc. 1966, 88, 4273. (b) Huang, D.-J.; Rayabarapu,

D. K.; Li, L.-P.; Sambaiah, T.; Cheng, C.-H. Chem. Eur. J. 2000, 6, 3706. (c) Jayanth, T. T. Jeganmohan, M.; Cheng, C.-H. J. Org. Chem. 2004, 69, 84. 


\section{Spectral Data of Compounds 4a-r}

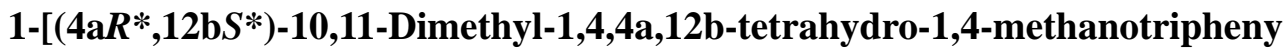

len-7-yl]ethanone (4a). Pale yellow solid; $\mathrm{R}_{\mathrm{f}}=0.75$ (3\% ethyl acetate in hexanes); IR $1675 \mathrm{~cm}^{-1}(\mathrm{C}=\mathrm{O}) ;{ }^{1} \mathrm{H} \mathrm{NMR}\left(\mathrm{CDCl}_{3}, 500 \mathrm{MHz}\right): \delta 8.47$ (d, $\left.J=1.5 \mathrm{~Hz}, 1 \mathrm{H}\right), 7.75$ (dd, $J=$ 7.5, 1.5 Hz, 1 H), 7.73 (s, 1 H), 7.37 (d, $J=7.5$ Hz, 1 H), 7.09 (s, 1 H), 6.37 - 6.33 (m, 2 H), 3.05 - 2.97 (m, 4 H), 2.63 (s, 3 H), 2.31 (s, 3 H), 2.27 (s, 3 H), 1.30 (d, J = $8.5 \mathrm{~Hz}, 1 \mathrm{H}), 1.14$ (d, $J=8.5 \mathrm{~Hz}, 1 \mathrm{H}) ;{ }^{13} \mathrm{C} \mathrm{NMR}\left(\mathrm{CDCl}_{3}, 125 \mathrm{MHz}\right): \delta$ 198.0, 143.4, 137.9, 137.3, 137.0, 135.3, 134.8, 132.7, 131.3, 130.3, 128.5, 127.0, 123.7, 122.0, 55.6, 55.2, 42.8, 41.6, 40.7, 26.6, 19.6, 19.5; HMRS (EI+): calcd for $\mathrm{C}_{23} \mathrm{H}_{22} \mathrm{O}$ 314.1671, found 314.1675.

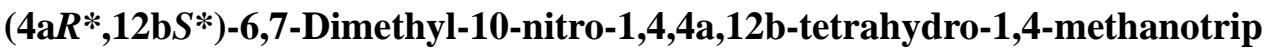

henyl-ene (4b). Pale yellow solid; $R_{\mathrm{f}}=0.70$ ( $2 \%$ ethyl acetate in hexanes); $\mathrm{IR} \mathrm{cm}^{-1}$ 1509, $1336\left(\mathrm{NO}_{2}\right) ;{ }^{1} \mathrm{H}$ NMR $\left(\mathrm{CDCl}_{3}, 400 \mathrm{MHz}\right): \delta 8.68$ (d, $\left.J=2.0 \mathrm{~Hz}, 1 \mathrm{H}\right), 7.99$ (dd, $J=8.4,2.4 \mathrm{~Hz}, 1 \mathrm{H}$ ), 7.65 (s, $1 \mathrm{H}$ ), 7.42 (d, $J=8.8 \mathrm{~Hz}, 1 \mathrm{H}$ ), 7.10 (s, $1 \mathrm{H}$ ), $6.38-6.33$ (m, 2 H), 3.05 - 2.96 (m, 4 H), 2.31 (s, 3 H), 2.28 (s, 3 H), 1.27 (d, $J=8.8$ Hz, 1 H), 1.15 (d, $J=8.8 \mathrm{~Hz}, 1 \mathrm{H}) ;{ }^{13} \mathrm{C} \mathrm{NMR}\left(\mathrm{CDCl}_{3}, 100 \mathrm{MHz}\right): \delta 147.0,145.5,138.4,138.3$, 137.5, 135.6, 135.4, 134.2, 131.6, 131.3, 127.6, 124.1, 121.7, 117.5, 56.0, 55.4, 43.0, 42.0, 41.0, 19.9, 19.8; HMRS (EI+): calcd for $\mathrm{C}_{21} \mathrm{H}_{19} \mathrm{NO}_{2}$ 317.1416, found 317.1420.

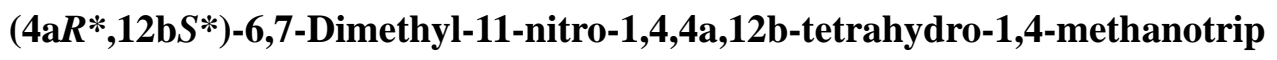
henyl-ene (4c). Pale yellow solid; $R_{\mathrm{f}}=0.72$ (2\% ethyl acetate in hexanes); IR 1524, $1351 \mathrm{~cm}^{-1}\left(\mathrm{NO}_{2}\right) ;{ }^{1} \mathrm{H}$ NMR $\left(\mathrm{CDCl}_{3}, 400 \mathrm{MHz}\right): \delta 8.15$ (d, $\left.J=2.0 \mathrm{~Hz}, 1 \mathrm{H}\right), 8.00$ (dd, $J$ = 8.4, $2.0 \mathrm{~Hz}, 1 \mathrm{H}), 7.94$ (d, $J=8.8 \mathrm{~Hz}, 1 \mathrm{H}), 7.64$ (s, $1 \mathrm{H}), 7.12$ (s, $1 \mathrm{H}), 6.37$ (s, $1 \mathrm{H})$, 6.63 (s, $1 \mathrm{H}), 3.08$ - 2.98 (m, 4 H), 2.30 (s, 3 H), 2.29 (s, 3 H), 1.27 (d, $J=8.8 \mathrm{~Hz}, 1$ H), 1.17 (d, $J=8.8 \mathrm{~Hz}, 1 \mathrm{H}) ;{ }^{13} \mathrm{C} \mathrm{NMR}\left(\mathrm{CDCl}_{3}, 100 \mathrm{MHz}\right): \delta$ 146.5, 139.1, 138.8, 138.8, 138.0, 137.3, 136.5, 135.1, 131. 5, 127.5, 125.3, 124.7, 122.9, 121.3, 55.9, 55.1, 42.7, 41.3, 40.8, 19.7, 19.6; HMRS (EI+): calcd for $\mathrm{C}_{21} \mathrm{H}_{19} \mathrm{NO}_{2} 317.1416$, found 317.1419. 


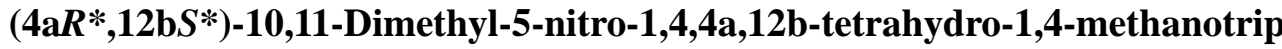
-henylene (4d). Pale yellow solid; $R_{f}=0.70$ (2\% ethyl acetate in hexanes); IR 1506, $1334 \mathrm{~cm}^{-1}\left(\mathrm{NO}_{2}\right) ;{ }^{1} \mathrm{H}$ NMR $\left(\mathrm{CDCl}_{3}, 400 \mathrm{MHz}\right): \delta 8.08$ (d, $\left.J=8.0 \mathrm{~Hz}, 1 \mathrm{H}\right), 7.61$ (dd, $J$ = 8.0, $1.2 \mathrm{~Hz}, 1 \mathrm{H}), 7.60(\mathrm{~s}, 1 \mathrm{H}), 7.29(\mathrm{t}, J=8.0 \mathrm{~Hz}, 1 \mathrm{H}), 7.09(\mathrm{~s}, 1 \mathrm{H}), 6.31(\mathrm{~s}, 1 \mathrm{H})$, 6.30 (s, $1 \mathrm{H}), 3.65$ (d, $J=9.6 \mathrm{~Hz}, 1 \mathrm{H}), 3.02$ (d, $J=9.6 \mathrm{~Hz}, 1 \mathrm{H}), 2.96$ (s, $1 \mathrm{H}), 2.72$ (s, $1 \mathrm{H}), 2.29$ (s, $3 \mathrm{H}), 2.28$ (s, $3 \mathrm{H}), 1.24$ (d, $J=8.8 \mathrm{~Hz}, 1 \mathrm{H}), 1.14$ (d, $J=8.8 \mathrm{~Hz}, 1$ $\mathrm{H}) ;{ }^{13} \mathrm{C}$ NMR $\left(\mathrm{CDCl}_{3}, 100 \mathrm{MHz}\right): \delta$ 151.2, 138.0, 137.8, 137.2, 135.1, 134.8, 134.7, 131.5, 131.1, 127.8, 126.5, 126.2, 124.1, 123.3, 55.0, 54.3, 43.0, 40.4, 37.1, 19.7, 19.5; HMRS (EI+): calcd for $\mathrm{C}_{21} \mathrm{H}_{19} \mathrm{NO}_{2}$ 317.1416, found 317.1417.

$\left(4 \mathrm{a} R^{*}, 12 \mathrm{~b} S^{*}\right)-6,7,10-T r i m e t h y l-1,4,4 \mathrm{a}, 12 \mathrm{~b}-$ tetrahydro-1,4-methanotriphenyl ene (4e). Colorless solid; $\mathrm{R}_{\mathrm{f}}=0.75$ (1\% ethyl acetate in hexanes); ${ }^{1} \mathrm{H}$ NMR $\left(\mathrm{CDCl}_{3}\right.$, $400 \mathrm{MHz}$ ): $\delta 7.67$ (s, 1 H), 7.66 (s, 1 H), 7.20 (d, $J=7.6$ Hz, 1 H), 7.10 (s, 1 H), 7.04 (dd, $J=7.6,1.2 \mathrm{~Hz}, 1 \mathrm{H}), 6.35$ (s, $1 \mathrm{H}), 6.34$ (d, $J=1.6 \mathrm{~Hz}, 1 \mathrm{H}), 2.99-2.95$ (m, $4 \mathrm{H}$ ), 2.39 (s, 3 H), 2.31 (s, 3 H), 2.28 (s, 3 H), 1.37 (d, $J=8.8$ Hz, 1 H), 1.14 (d, $J=8.5$ Hz, $1 \mathrm{H}) ;{ }^{13} \mathrm{C} \mathrm{NMR}\left(\mathrm{CDCl}_{3}, 100 \mathrm{MHz}\right): \delta 137.5,137.4,136.0,135.4,135.3,134.7,134.3$, 131.9, 131.3, 130.0, 129.6, 128.0, 123.4, 122.6, 55.4, 55.3, 42.8, 40.8, 40.7, 21.4, 19.7, 19.5; HMRS (EI+): calcd for $\mathrm{C}_{22} \mathrm{H}_{22}$ 286.1721, found 286.1720.

(4aR*,12bS*)-6,7,11-Trimethyl-1,4,4a,12b-tetrahydro-1,4-methanotriphenyl ene (4f). Colorless solid; $\mathrm{R}_{\mathrm{f}}=0.75$ (1\% ethyl acetate in hexanes); ${ }^{1} \mathrm{H}$ NMR $\left(\mathrm{CDCl}_{3}\right.$, $400 \mathrm{MHz}$ ): $\delta 7.78$ (d, $J=8.0 \mathrm{~Hz}, 1 \mathrm{H}), 7.64$ (s, $1 \mathrm{H}), 7.14$ (s, $1 \mathrm{H}), 7.10$ (s, $1 \mathrm{H}), 7.03$ (d, $J=8.0 \mathrm{~Hz}, 1 \mathrm{H}), 6.36$ (s, $2 \mathrm{H}), 3.00$ (s, 4 H), 2.37 (s, 3 H), 2.31 (s, $3 \mathrm{H}), 2.29$ (s, 3 H), $1.40(\mathrm{~d}, J=8.8 \mathrm{~Hz}, 1 \mathrm{H}), 1.17(\mathrm{~d}, J=8.4 \mathrm{~Hz}, 1 \mathrm{H}) ;{ }^{13} \mathrm{C} \mathrm{NMR}\left(\mathrm{CDCl}_{3}, 100 \mathrm{MHz}\right)$ : $\delta$ 137.6, 137.5, 137.4, 136.7, 135.7, 135.0, 134.3, 131.2, 131.7, 129.6, 129.5, 127.1, 123.3, 122.0, 55.4, 55.3, 43.0, 41.2, 40.7, 21.1, 19.7, 19.4; HMRS (EI): calcd for $\mathrm{C}_{22} \mathrm{H}_{22}$ 286.1722, found 286.1717.

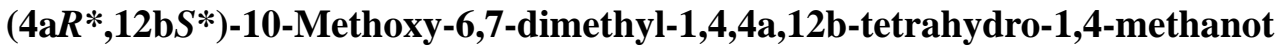


riphenylene (4g). Colorless Solid; $\mathrm{R}_{\mathrm{f}}=0.65$ (2\% ethyl acetate in hexanes); ${ }^{1} \mathrm{H}$ NMR $\left(\mathrm{CDCl}_{3}, 400 \mathrm{MHz}\right): \delta 7.59$ (s, $\left.1 \mathrm{H}\right), 7.37$ (d, $\left.J=2.4 \mathrm{~Hz}, 1 \mathrm{H}\right), 7.21$ (d, $\left.J=8.4 \mathrm{~Hz}, 1 \mathrm{H}\right)$, 7.09 (s, $1 \mathrm{H}$ ), 6.80 (dd, $J$ = 8.4, 2.4 Hz, $1 \mathrm{H}$ ), 6.33 (s, $1 \mathrm{H}), 6.32$ (s, $1 \mathrm{H}$ ), 3.86 (s, $3 \mathrm{H}$ ), 2.99 (s, 2 H), 2.95 (s, 1 H), 2.91 (s, 1 H), 2.29 (s, 3 H), 2.27 (s, 3 H), 1.34 (d, $J=8.8$ $\mathrm{Hz}, 1 \mathrm{H}), 1.47$ (d, $J=8.8 \mathrm{~Hz}, 1 \mathrm{H}) ;{ }^{13} \mathrm{C} \mathrm{NMR}\left(\mathrm{CDCl}_{3}, 100 \mathrm{MHz}\right): \delta$ 158.0,137.5, 137.4, 136.4, 135.6, 134.3, 133.2, 131.3, 131.0, 130.2, 129.4, 123.5, 113.0, 107.3, 55.4, 55.3, 55.2, 42.8, 40.8, 40.5, 19.7, 19.5; HMRS (EI+): calcd for $\mathrm{C}_{22} \mathrm{H}_{22} \mathrm{O}$ 302.1671, found 302.1669.

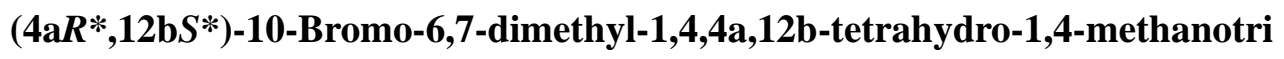
phenylene (4h). Colorless solid; $\mathrm{R}_{\mathrm{f}}=0.70$ (1\% ethyl acetate in hexanes); ${ }^{1} \mathrm{H}$ NMR $\left(\mathrm{CDCl}_{3}, 400 \mathrm{MHz}\right): \delta 7.96$ (d, $\left.J=2.0 \mathrm{~Hz}, 1 \mathrm{H}\right), 7.55$ (s, $\left.1 \mathrm{H}\right), 7.29$ (dd, $J=8.0,2.4 \mathrm{~Hz}$, $1 \mathrm{H}), 7.15$ (d, $J=8.4 \mathrm{~Hz}, 1 \mathrm{H}), 7.08$ (s, $1 \mathrm{H}), 6.34-6.32$ (m, 2 H), 2.96 - 2.91 (m, 4 H), 2.29 (s, $3 \mathrm{H}), 2.28$ (s, $3 \mathrm{H}), 1.31$ (d, $J=8.8 \mathrm{~Hz}, 1 \mathrm{H}), 1.14$ (d, $J=8.8 \mathrm{~Hz}, 1 \mathrm{H}) ;{ }^{13} \mathrm{C}$ NMR $\left(\mathrm{CDCl}_{3}, 100 \mathrm{MHz}\right): \delta$ 137.7, 137.3, 137.0, 136.6, 135.3, 134.6, 134.4, 131.7, 131.3, 129.8, 128.2, 125.0, 123.6, 120.1, 55.4, 55.2, 42.7, 40.9, 40.7, 19.7, 19.5; HMRS (EI+): calcd for $\mathrm{C}_{21} \mathrm{H}_{19} \mathrm{Br} 350.0670$, found 350.0673.

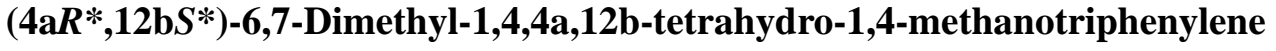

(4i). Colorless solid; $\mathrm{R}_{\mathrm{f}}=0.70$ (1\% ethyl acetate in hexanes); ${ }^{1} \mathrm{H}$ NMR $\left(\mathrm{CDCl}_{3}, 400\right.$ MHz): $\delta 7.91$ (dd, $J=6.4,2.8 \mathrm{~Hz}, 1 \mathrm{H}), 7.68$ (s, $1 \mathrm{H}), 7.32$ (dd, $J=5.6,2.0 \mathrm{~Hz}, 1 \mathrm{H}$ ), 7.23 - 7.21 (m, 2 H), 7.12 (s, 1 H), 6.38 - 6.37 (m, 2 H), 3.05 - 3.00 (m, 4 H), 2.32 (s,

$3 \mathrm{H}), 2.30$ (s, $3 \mathrm{H}), 1.40$ (d, $J=8.8 \mathrm{~Hz}, 1 \mathrm{H}), 1.18$ (d, $J=8.8 \mathrm{~Hz}, 1 \mathrm{H}) ;{ }^{13} \mathrm{C}$ NMR $\left(\mathrm{CDCl}_{3}, 100 \mathrm{MHz}\right): \delta 137.6,137.5,137.4,136.2,135.3,134.3,132.1,131.3,130.1$, 129.5, 127.1, 126.1, 123.5, 122.0, 55.5, 55.3, 42.8, 41.2, 40.7, 19.7, 19.5; HMRS (EI+): calcd for $\mathrm{C}_{21} \mathrm{H}_{20} 272.1565$, found 272.1569.

1-[(4a $\left.R^{*}, 12 \mathrm{~b} S^{*}\right)-6,7-D i m e t h y l-8 b, 9,14,14 a-t e t r a h y d r o-9,14-e p o x y b e n z o[f]$ tet raphen-3-yl)ethanone $(\mathbf{4 j})$. Pale yellow solid; $R_{f}=0.60$ (8\% ethyl acetate in 
hexanes); IR $1675 \mathrm{~cm}^{-1}(\mathrm{C}=\mathrm{O}) ;{ }^{1} \mathrm{H}$ NMR $\left(\mathrm{CDCl}_{3}, 500 \mathrm{MHz}\right): \delta 8.51$ (d, $J=1.5 \mathrm{~Hz}, 1$ H), 7.83 (d, $J=7.5 \mathrm{~Hz}, 1 \mathrm{H}), 7.70$ (s, $1 \mathrm{H}), 7.48$ (d, $J=8.5 \mathrm{~Hz}, 1 \mathrm{H}), 7.45-7.41$ (m, 2 H), 7.26 - 7.22 (m, 2 H), 7.18 (s, 1 H), 5.37 (s, 2 H), 3.40 (d, J = 9.5 Hz, 1 H), 3.35 (d, $J=9.5 \mathrm{~Hz}, 1 \mathrm{H}), 2.65$ (s, $3 \mathrm{H}), 2.33$ (s, $3 \mathrm{H}), 2.32$ (s, $3 \mathrm{H}):{ }^{13} \mathrm{C} \mathrm{NMR}\left(\mathrm{CDCl}_{3}, 125\right.$ MHz); $\delta 198.0,145.7,145.5,140.4,137.5,135.7,132.7,132.5,131.1,130.2,128.4$ 127.3, 127.1, 127.0, 124.1, 122.4, 119.3, 119.2, 90.2, 44.3, 43.5, 26.7, 19.7, 19.6; HMRS (EI+): calcd for $\mathrm{C}_{26} \mathrm{H}_{22} \mathrm{O}_{2}$ 366.1620, found 366.1615.

2,3-[(4a $\left.R^{*}, 12 \mathrm{~b} S *\right)$-Dimethyl-8b,9,14,14a-tetrahydro-9,14-epoxybenzo[f]tetra phene (4k). Colorless solid; $\mathrm{R}_{\mathrm{f}}=0.65$ (7\% ethyl acetate in hexanes); ${ }^{1} \mathrm{H}$ NMR $\left(\mathrm{CDCl}_{3}, 400 \mathrm{MHz}\right): \delta 7.90(\mathrm{~d}, J=7.6 \mathrm{~Hz}, 1 \mathrm{H}), 7.68(\mathrm{~s}, 1 \mathrm{H}), 7.44-7.37$ (m, $\left.3 \mathrm{H}\right)$, 7.28 - 7.23 (m, 4 H), 7.22 (s, 1 H), 5.37 (s, 2 H), 3.37 (d, $J=9.6 \mathrm{~Hz}, 1 \mathrm{H}), 3.33$ (d, $J$ $=9.2 \mathrm{~Hz}, 1 \mathrm{H}), 2.31(\mathrm{~s}, 3 \mathrm{H}), 2.30(\mathrm{~s}, 3 \mathrm{H}) ;{ }^{13} \mathrm{C} \mathrm{NMR}\left(\mathrm{CDCl}_{3}, 100 \mathrm{MHz}\right): \delta 145.8$, 145.7, 136.6, 134.8, 132.3, 132.1, 130.9, 129.9, 129.4, 127.5, 126.9, 123.9, 122.4, 119.2, 119.1, 90.3, 90.2, 43.9, 43.5, 19.7, 19.5; HMRS (EI+): calcd for $\mathrm{C}_{24} \mathrm{H}_{20} \mathrm{O}$ 324.1514, found 324.1518.

\section{8,9-[(4a $\left.R^{*}, 12 \mathrm{~b} S *\right)$-Dimethyl-10b,11,16,16a-tetrahydro-11,16-epoxydibenzo[a}

,fltetraphene (4I). Colorless solid; $\mathrm{R}_{\mathrm{f}}=0.70$ (7\% ethyl acetate in hexanes); ${ }^{1} \mathrm{H}$ NMR $\left(\mathrm{CDCl}_{3}, 400 \mathrm{MHz}\right): \delta 8.10$ (d, $\left.J=9.2 \mathrm{~Hz}, 1 \mathrm{H}\right), 7.85$ (d, $\left.J=8.0 \mathrm{~Hz}, 1 \mathrm{H}\right), 7.79$ (s, $1 \mathrm{H}$ ), 7.77 (d, $J=8.0$ Hz, 1 H), 7.65 - 7.59 (m, 2 H), 7.52 - 7.48 (m, 2 H), 7.35 - 7.31 (m, 2 H), 7.25 (s, $1 \mathrm{H}), 5.50$ (s, $1 \mathrm{H}), 5.41$ (s, $1 \mathrm{H}), 3.92$ (d, $J=9.6 \mathrm{~Hz}, 1 \mathrm{H}), 3.52$ (d, $J=9.6$ Hz, 1 H), 2.34 (s, 3 H), 2.31 (s, 3 H); $\left.{ }^{13} \mathrm{C} \mathrm{NMR} \mathrm{(CDCl} 3,100 \mathrm{MHz}\right): \delta$ 146.5, 145.3, 136.8, 135.2, 133.4, 132.5, 132.2, 130.7, 130.1, 129.4, 129.1, 128.9, 127.6, 127.1, 127.0, 126.7, 125.2, 124.6, 123.4, 121.2, 119.7, 118.5, 90.6, 88.5, 43.3, 41.5, 19.8, 19.5; HMRS (EI+): calcd for $\mathrm{C}_{28} \mathrm{H}_{22} 0$ 374.1671, found 374.1668.

5,6-[(4a $\left.R^{*}, 12 \mathrm{~b} S *\right)$-Dimethyl-7b,8,13,13a-tetrahydro-8,13-epoxytetrapheno[6 ,5-b]thiophene (4m). Colorless Solid; $\mathrm{R}_{\mathrm{f}}=0.70$ (7\% ethyl acetate in hexanes); ${ }^{1} \mathrm{H}$ 
NMR (CDCl $3,400 \mathrm{MHz}): \delta 7.43-7.39$ (m, $2 \mathrm{H}), 7.32$ (d, $J=7.2 \mathrm{~Hz}, 1 \mathrm{H}), 7.31$ (s, 1 H), 7.25 - 7.22 (m, 2 H), 7.14 (s, $1 \mathrm{H}), 5.45$ (s, $1 \mathrm{H}), 5.41$ (s, $1 \mathrm{H}), 3.51$ (d, $J=9.6 \mathrm{~Hz}$, $1 \mathrm{H}), 3.41$ (d, J = 9.6 Hz, $1 \mathrm{H}), 2.27$ (s, $3 \mathrm{H}), 2.26$ (s, $3 \mathrm{H}) ;{ }^{13} \mathrm{C} \mathrm{NMR}\left(\mathrm{CDCl}_{3}, 100\right.$ MHz): $\delta 145.9,145.3,136.6,135.7,135.4,134.1,131.4,130.8,128.5,127.0,126.9$, 124.4, 124.0, 122.8, 119.8, 119.3, 90.1, 88.9, 45.3, 41.5, 19.6, 19.4; HMRS (EI): calcd for $\mathrm{C}_{22} \mathrm{H}_{18} \mathrm{OS} 330.1078$, found 330.1083.

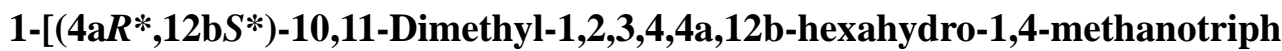
enylen-7-yl)ethano-ne (4n). Colorless Solid; $R_{\mathrm{f}}=0.75$ (2\% ethyl acetate in hexanes); IR $1674 \mathrm{~cm}^{-1}(\mathrm{C}=\mathrm{O}) ;{ }^{1} \mathrm{H}$ NMR $\left(\mathrm{CDCl}_{3}, 400 \mathrm{MHz}\right): \delta 8.41$ (s, $\left.1 \mathrm{H}\right), 7.71$ (dd, $J=8.0$, $1.6 \mathrm{~Hz}, 1 \mathrm{H}), 7.67$ (s, $1 \mathrm{H}), 7.26$ (d, $J=8.0 \mathrm{~Hz}, 1 \mathrm{H}), 6.99$ (s, $1 \mathrm{H}), 3.18$ (d, $J=10.0$ Hz, 1 H), 3.13 (d, J = 10.0 Hz, 1 H), 2.61 (s, 3 H), 2.60 (s, 1 H), 2.29 (s, 3 H), 2.24 (s, $3 \mathrm{H}), 1.70-1.62$ (m, $2 \mathrm{H}), 1.32$ (d, $J=10.0 \mathrm{~Hz}, 1 \mathrm{H}), 1.01$ (dd, $J=10.4,1.6 \mathrm{~Hz}, 1 \mathrm{H}$ );

${ }^{13} \mathrm{C} \mathrm{NMR}\left(\mathrm{CDCl}_{3}, 100 \mathrm{MHz}\right): \delta$ 198.0, 143.2, 136.9, 135.2, 134.9, 134.7, 132.1, 131.2, 130.3, 127.9, 126.9, 123.4, 121.6, 49.7, 49.2, 46.1, 45.3, 33.2, 30.2, 30.1, 26.5, 19.5, 19.4; HMRS (EI+): calcd for $\mathrm{C}_{23} \mathrm{H}_{24} \mathrm{O} 316.1827$, found 316.1822 .

1-[(4a $\left.\left.R^{*}, 12 \mathrm{~b} S^{*}\right)-1,4,4 \mathrm{a}, 12 \mathrm{~b}-T e t r a h y d r o-1,4-m e t h a n o t r i p h e n y l e n-7-y l\right) e t h a n o$ ne (4o). Pale yellow Solid; $R_{\mathrm{f}}=0.70$ (2\% ethyl acetate in hexanes); IR $1675 \mathrm{~cm}^{-1}$ $(\mathrm{C}=\mathrm{O}) ;{ }^{1} \mathrm{H} \mathrm{NMR}\left(\mathrm{CDCl}_{3}, 500 \mathrm{MHz}\right): \delta 8.50$ (d, $\left.J=1.5 \mathrm{~Hz}, 1 \mathrm{H}\right), 7.97$ (d, $J=9.5 \mathrm{~Hz}$, $1 \mathrm{H}$ ), 7.77 (dd, $J=7.5,1.5 \mathrm{~Hz}, 1 \mathrm{H}$ ), 7.38 (d, $J=8.0 \mathrm{~Hz}, 1 \mathrm{H}), 7.33$ (t, $J=2.0 \mathrm{~Hz}, 1$ H), 7.27 - 7.25 (m, 2 H), 6.37 - 6.36 (m, 2 H), 3.06 (s, 2 H), 2.99 - 2.98 (m, 2 H), $2.63(\mathrm{~s}, 3 \mathrm{H}), 1.31(\mathrm{~d}, J=8.5 \mathrm{~Hz}, 1 \mathrm{H}), 1.15(\mathrm{~d}, J=8.5 \mathrm{~Hz}, 1 \mathrm{H}) ;{ }^{13} \mathrm{C}$ NMR $\left(\mathrm{CDCl}_{3}\right.$, 125 MHz); $\delta$ 197.8, 143.5, 137.9, 137.8, 137.5, 135.4, 132.4, 130.9, 130.4, 128.3, 127.4, 126.5, 122.7, 122.5, 55.6, 55.4, 42.7, 41.6, 41.3, 26.5; HMRS (EI+): calcd for $\mathrm{C}_{21} \mathrm{H}_{18} \mathrm{O}$ 286.1358, found 286.1360.

$1-\left[\left(4 \mathrm{a} R^{*}, 12 \mathrm{~b} S^{*}\right)-4,4 \mathrm{a}, 10,11,12,13 \mathrm{~b}-H e x a h y d r o-1 H-1,4-\right.$ methanocyclopenta[b] triphenylen-7-yl)ethanone (4p). Pale yellow Solid; $R_{\mathrm{f}}=0.75$ (2\% ethyl acetate in 
hexanes); IR $1677 \mathrm{~cm}^{-1}(\mathrm{C}=\mathrm{O}) ;{ }^{1} \mathrm{H} \mathrm{NMR}\left(\mathrm{CDCl}_{3}, 400 \mathrm{MHz}\right): \delta 8.48(\mathrm{~d}, J=2.0 \mathrm{~Hz}$, $1 \mathrm{H}$ ), 7.85 (s, $1 \mathrm{H}$ ), 7.77 (dd, $J=8.0,1.6 \mathrm{~Hz}, 1 \mathrm{H}), 7.37$ (d, $J=8.0 \mathrm{~Hz}, 1 \mathrm{H}$ ), 7.19 (s, 1 H), 6.36 - 6.34 (m, 2 H), 3.03 (s, 2 H), 2.97 - 2.90 (m, 6 H), 2.63 (s, 3 H), $2.11-2.07$ (m, $2 \mathrm{H}), 1.32$ (d, $J=8.8 \mathrm{~Hz}, 1 \mathrm{H}), 1.13$ (d, $J=8.8 \mathrm{~Hz}, 1 \mathrm{H}):{ }^{13} \mathrm{C} \mathrm{NMR}\left(\mathrm{CDCl}_{3}, 100\right.$ MHz); $\delta 198.0,144.8,143.4,142.8,137.9,137.3,135.9,135.2,133.0,130.3,128.9$, 126.9, 125.9, 122.2, 118.4, 55.6, 55.5, 42.7, 41.6, 41.4, 32.6, 26.6, 25.4; HMRS (EI+): calcd for $\mathrm{C}_{24} \mathrm{H}_{22} \mathrm{O} 326.1671$, found 326.1670.

\section{$1-\left[\left(4 a R^{*}, 12 b S^{*}\right)-1,4,4 a, 13 b-T e t r a h y d r o-1,4-m e t h a n o t r i p h e n y l e n o[2,3-d][1,3]\right.$}

dioxol-7-yl)ethanone (4q). Pale yellow Solid; $\mathrm{R}_{\mathrm{f}}=0.75$ (2\% ethyl acetate in hexanes); IR $1675 \mathrm{~cm}^{-1}(\mathrm{C}=\mathrm{O}) ;{ }^{1} \mathrm{H}$ NMR $\left(\mathrm{CDCl}_{3}, 400 \mathrm{MHz}\right): \delta 8.29$ (s, $\left.1 \mathrm{H}\right), 7.75$ (dd, $J=7.2$, $2.8 \mathrm{~Hz}, 1 \mathrm{H}), 7.42$ (s, $1 \mathrm{H}), 7.36$ (d, J = $8.0 \mathrm{~Hz}, 1 \mathrm{H}), 6.76$ (s, $1 \mathrm{H}), 6.34$ (s, $2 \mathrm{H}), 5.96$ (s, 2 H), 3.05 - 2.96 (m, 4 H), 2.61 (s, 3 H), 1.24 (d, $J=9.4$ Hz, 1 H), 1.15 (d, J = 8.8

$\mathrm{Hz}, 1 \mathrm{H}) ;{ }^{13} \mathrm{C} \mathrm{NMR}\left(\mathrm{CDCl}_{3}, 100 \mathrm{MHz}\right): \delta$ 198.2, 147.0, 142.8, 137.8, 137.5, 135.6, 132.6, 132.3, 130.7, 128.3, 126.8, 120.9, 109.4, 102.6, 101.2, 55.7, 55.2, 42.7, 41.7, 41.6, 22.6; HMRS (EI+): calcd for $\mathrm{C}_{22} \mathrm{H}_{18} \mathrm{O}_{3} 330.1253$, found 330.1257.

1-[(4a $\left.R^{*}, 12 \mathrm{~b} S *\right)-10-M e t h y l-1,4,4 a, 12 b-t e t r a h y d r o-1,4-m e t h a n o t r i p h e n y l e n-7$ yl)ethanone and 1-[(4a $\left.R^{*}, 12 \mathrm{~b} S^{*}\right)-11-M e t h y l-1,4,4 \mathrm{a}, 12 \mathrm{~b}-$-tetrahydro-1,4-methano triphenylen-7-yl)ethanone (4r and $4 \mathbf{r}^{\prime}$ ). Pale yellow Solid; $R_{\mathrm{f}}=0.70$ (2\% ethyl acetate in hexanes); IR $1675 \mathrm{~cm}^{-1}(\mathrm{C}=\mathrm{O}) ;{ }^{1} \mathrm{H} \mathrm{NMR}\left(\mathrm{CDCl}_{3}, 400 \mathrm{MHz}\right): \delta 8.49$ (d, $J=$ $1.6 \mathrm{~Hz}, 1 \mathrm{H}), 8.46$ (d, $J=1.6 \mathrm{~Hz}, 1 \mathrm{H}), 7.86$ (d, $J=8.0 \mathrm{~Hz}, 1 \mathrm{H}), 7.79-7.75$ (m, $3 \mathrm{H})$, 7.39 - 7.37 (m, 2 H), 7.21 (d, $J=7.6 \mathrm{~Hz}, 1 \mathrm{H}), 7.14$ (s, $1 \mathrm{H}), 7.08$ - 7.05 (m, $2 \mathrm{H}$ ), 6.36 - 6.35 (m, 4 H), 3.06 - 2.96 (m, 8 H), 2.63 (s, 3 H), 2.62 (s, 3 H), 2.39 (s, 3 H), 2.35 (s, $3 \mathrm{H}), 1.31$ - 1.28 (m, $2 \mathrm{H}), 1.15$ - 1.13 (m, $2 \mathrm{H}) ;{ }^{13} \mathrm{C}$ NMR $\left(\mathrm{CDCl}_{3}, 100\right.$ MHz): $\delta$ 197.9, 143.2, 142.8, 137.9, 137.7, 137.4, 137.3, 135.9, 135.2, 134.9, 132.5, 132.4, 130.7, 130.6, 130.4, 130.3, 130.0, 129.3, 128.2, 127.5, 127.4, 126.9, 123.1, 122.6, 122.3, 55.6, 55.5, 55.3, 55.2, 42.8, 42.7, 41.6, 41.2, 40.8, 26.6, 21.3, 21.1; 
HMRS (EI+): calcd for $\mathrm{C}_{22} \mathrm{H}_{20} \mathrm{O} 300.1514$, found 300.1515 .

Procedure for Deoxyaromatization of $4 \mathrm{j}$ to Benzo[b]triphenylenes (7a). A round-bottom sidearm flask (25 mL) containing $\mathbf{4 j}$ (1.00 mmol) was evacuated and purged with nitrogen gas three times. Dichloromethane $(3.0 \mathrm{~mL})$ and $\mathrm{BF}_{3} . \mathrm{OEt}_{2}(1.50$ mmol) were added. The reaction mixture was stirred at room temperature for $1 \mathrm{~h}$. The reaction mixture was then poured into a saturated aqueous $\mathrm{NaHCO}_{3}$ solution and extracted with dichloromethane. The organic layer was washed with brine, dried with $\mathrm{MgSO}_{4}$, and concentrated under reduced pressure. The residue was recrystallized from a mixture of dichloromethane and hexane (1:3) to afford the desired product 7a. Product $\mathbf{7 b}$ was also prepared according to this procedure using $\mathbf{4 k}$ as the substrate.

1-(6,7-Dimethylbenzo[f]tetraphen-3-yl)ethanone (7a). Colorless Solid; m.p. $276-27{ }^{\circ} \mathrm{C}$, IR $1675 \mathrm{~cm}^{-1}(\mathrm{C}=\mathrm{O}),{ }^{1} \mathrm{H}$ NMR $\left(\mathrm{CDCl}_{3}, 500 \mathrm{MHz}\right): \delta 9.08$ (d, $J=1.5$ Hz, 1 H), 9.01 (s, 1 H), 8.90 (s, 1 H), 8.72 (d, $J$ = 9.0, Hz, 1 H), 8.43 (s, 1 H), 8.32 (s, $1 \mathrm{H}), 8.10$ (dd, $J=9.5,1.5 \mathrm{~Hz}, 2 \mathrm{H}), 8.05$ (d, $J=8.0 \mathrm{~Hz}, 2 \mathrm{H}), 7.58-7.53$ (m, $2 \mathrm{H}$ ), 2.76 (s, $3 \mathrm{H}), 2.52$ (s, $3 \mathrm{H}), 2.51$ (s, $3 \mathrm{H}) ;{ }^{13} \mathrm{C} \mathrm{NMR}\left(\mathrm{CDCl}_{3}, 125 \mathrm{MHz}\right): \delta$ 198.1, 137.1, 136.9, 135.5, 133.7, 132.8, 131.9, 130.0, 128.8, 128.3, 128.2, 128.0, 127.5, 127.4, 126.6, 126.2, 126.1, 124.3, 124.2, 123.9, 123.6, 123.2, 121.7, 26.6, 20.3, 20.2; HMRS (EI+): calcd for $\mathrm{C}_{26} \mathrm{H}_{20} \mathrm{O} 348.1514$, found 348.1520.

2,3-Dimethylbenzo[f]tetraphene (7b). Colorless Solid; m.p. $270-271{ }^{\circ} \mathrm{C},{ }^{1} \mathrm{H}$ NMR (CDCl $3,400 \mathrm{MHz}): \delta 9.01$ (s, $1 \mathrm{H}), 8.98$ (s, $1 \mathrm{H}), 8.71$ (dd, $J=6.0,3.6 \mathrm{~Hz}, 1 \mathrm{H}$ ), 8.50 (dd, $J=6.0,3.2 \mathrm{~Hz}, 1 \mathrm{H}), 8.44$ (s, $1 \mathrm{H}), 8.26$ (s, $1 \mathrm{H}), 8.04$ (d, $J=6.8 \mathrm{~Hz}, 2 \mathrm{H})$, 7.60 - 7.52 (m, $4 \mathrm{H}), 2.50$ (s, $3 \mathrm{H}), 2.48$ (s, $3 \mathrm{H}) ;{ }^{13} \mathrm{C}$ NMR $\left(\mathrm{CDCl}_{3}, 100 \mathrm{MHz}\right): \delta$ 136.5, 136.3, 132.2, 131.9, 130.1, 129.8, 128.5, 128.4, 128.1, 128.0, 127.9, 127.4, 126.9, 125.9, 125.7, 124.3, 124.1, 123.6, 123.1, 121.9, 121.5, 20.2; HMRS (EI+): calcd for $\mathrm{C}_{24} \mathrm{H}_{18} 306.1409$, found 306.1403. 
Procedure for the Isolation of Intermediate $C\left(R=H\right.$ and $\left.L=P P h_{3}\right)$. A round-bottom side-arm flask containing $\mathrm{Pd}\left(\mathrm{PPh}_{3}\right)_{4}(1.00 \mathrm{mmol})$, iodobenzene (1.00 mmol) and CsF (1.50 mmol) was purged with nitrogen gas three times. To the flask were added $\mathrm{CH}_{3} \mathrm{CN}(6 \mathrm{~mL})$ and norbornadiene $(5.00 \mathrm{mmol})$, and the solution was stirred at ambient temperature for $6 \mathrm{~h}$. At the end of the reaction, the solvent and the unreacted norbomadiene were removed in vacuo, and the residue was then washed with ether three times and ether layer was concentrated to give orthometallation intermediate $\mathbf{C}\left(\mathrm{R}=\mathrm{H}\right.$ and $\left.\mathrm{L}=\mathrm{PPh}_{3}\right)$ in $69 \%$ yield.

The ${ }^{1} \mathrm{H}$ NMR spectrum of intermediate $\mathbf{C}$ was compared with our previous report. ${ }^{1}$ In our previous report, ${ }^{1}$ we have synthesized the intermediate $\mathbf{C}$ and characterized it very well.

Further, intermediate C $(0.500 \mathrm{mmol})$ and CsF $(0.750 \mathrm{mmol})$ was taken in $25 \mathrm{~mL}$ round-bottom flask and purged with nitrogen gas three times. To the flask were added $\mathrm{CH}_{3} \mathrm{CN}$ (3 $\mathrm{mL}$ ) and benzyne precursor (3a) (0.600 mmol), and the solution was stirred at ambient temperature for $6 \mathrm{~h}$. At the end of the reaction, the reaction mixture was diluted with $\mathrm{CH}_{2} \mathrm{Cl}_{2}$, filtered through Celite and silica gel, and the filtrate was concentrated. The crude residue was purified through a silica gel column using hexanes and ethyl acetate as eluent to give pure $\mathbf{4 i}$ in $75 \%$ yield.

\section{References}

1. Liu, C.-H.; Li, C.-S.; Cheng, C.-H. Organometallics 1994, 13, 18. 
${ }^{1} \mathrm{H}$ and ${ }^{13} \mathrm{C}$ NMR Spectra of Compound 4a.
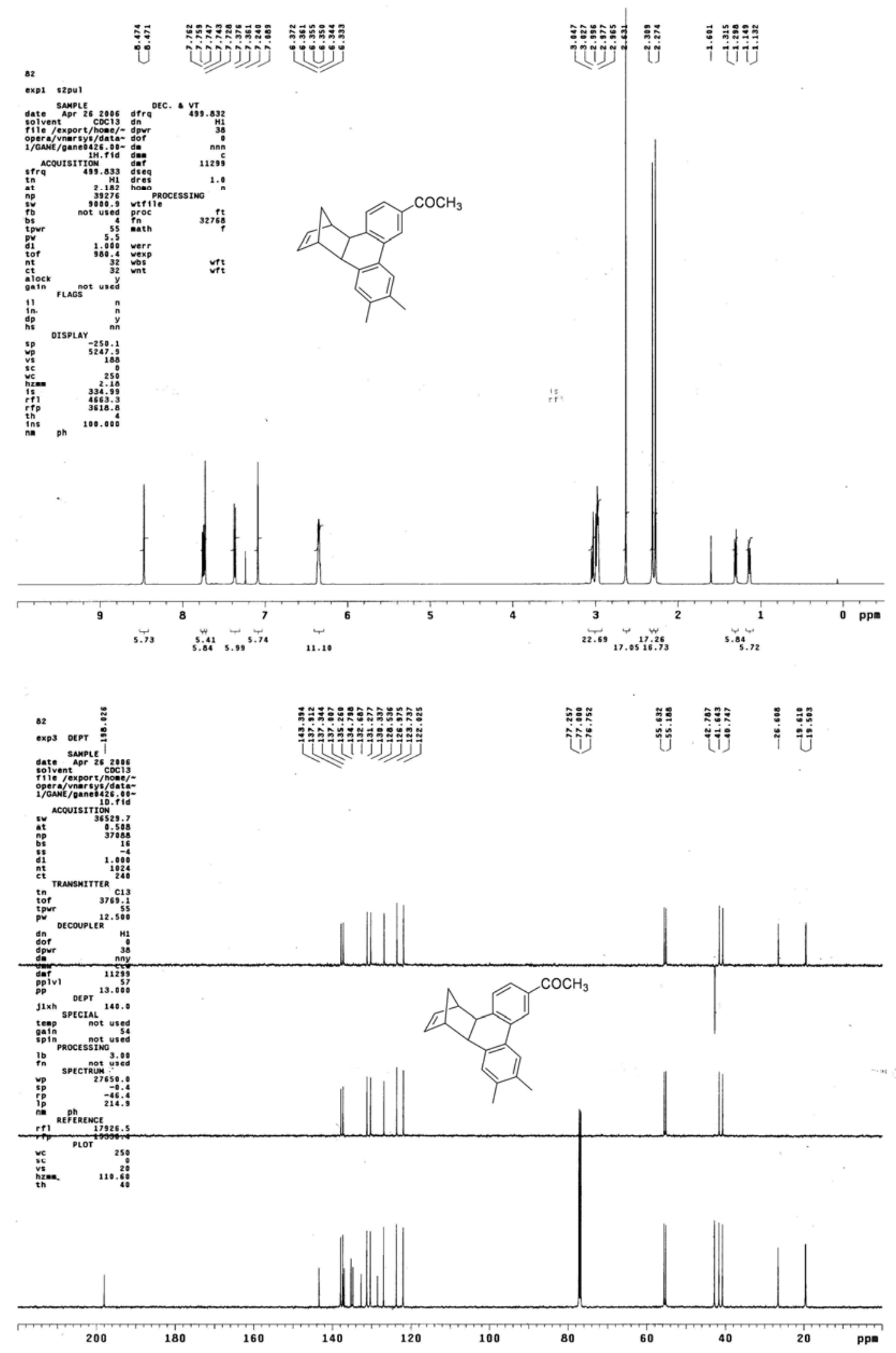
${ }^{1} \mathrm{H}$ and ${ }^{13} \mathrm{C}$ NMR Spectra of Compound $\mathbf{4 b}$.
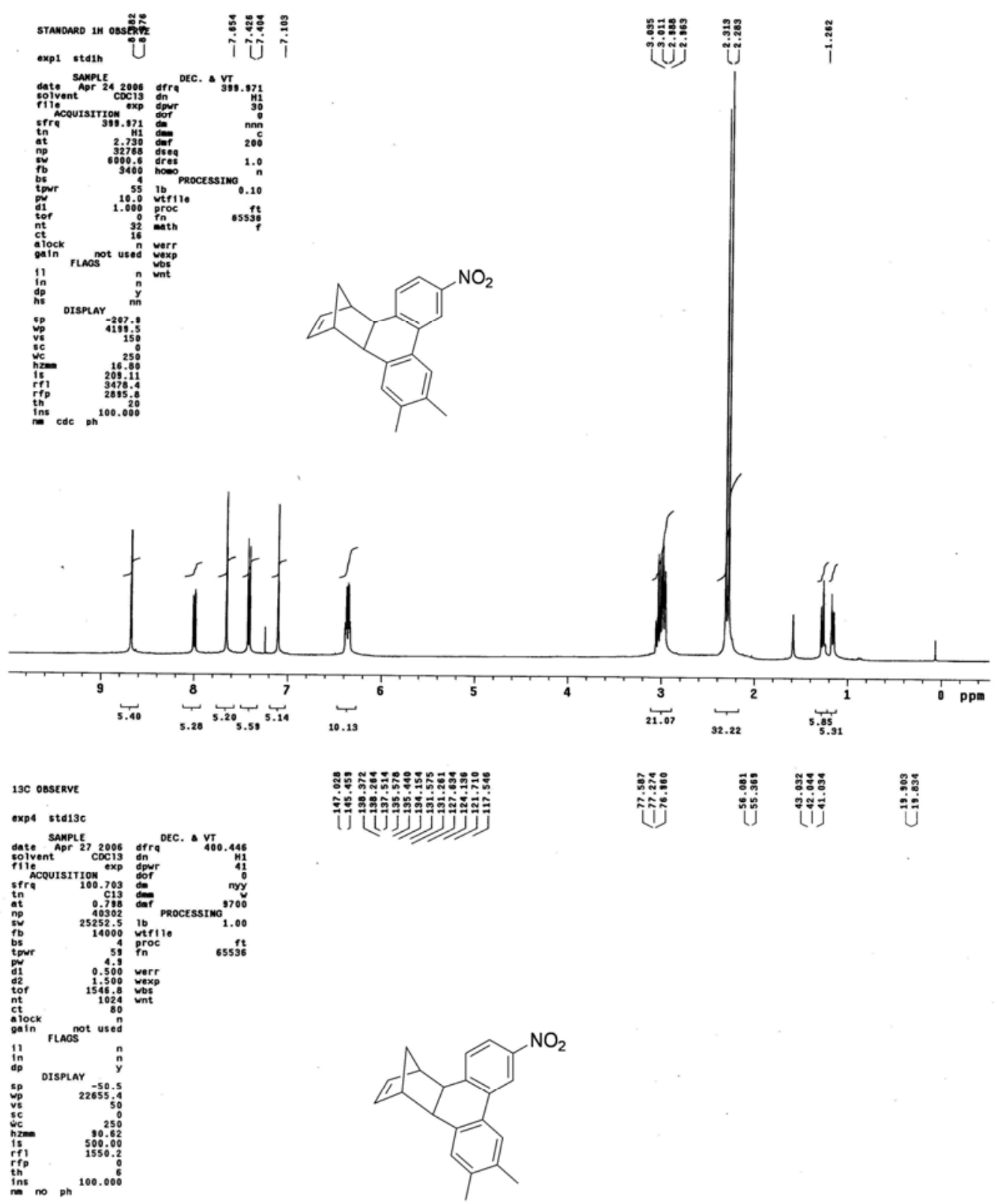

\begin{tabular}{|c|c|c|}
\hline & 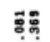 & ตุว \\
\hline 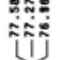 & 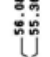 & ஸ் \\
\hline
\end{tabular}

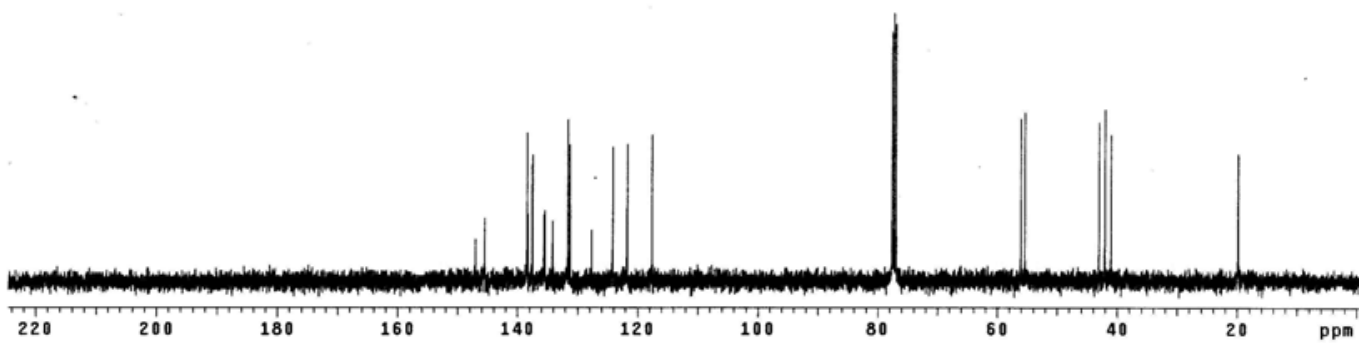



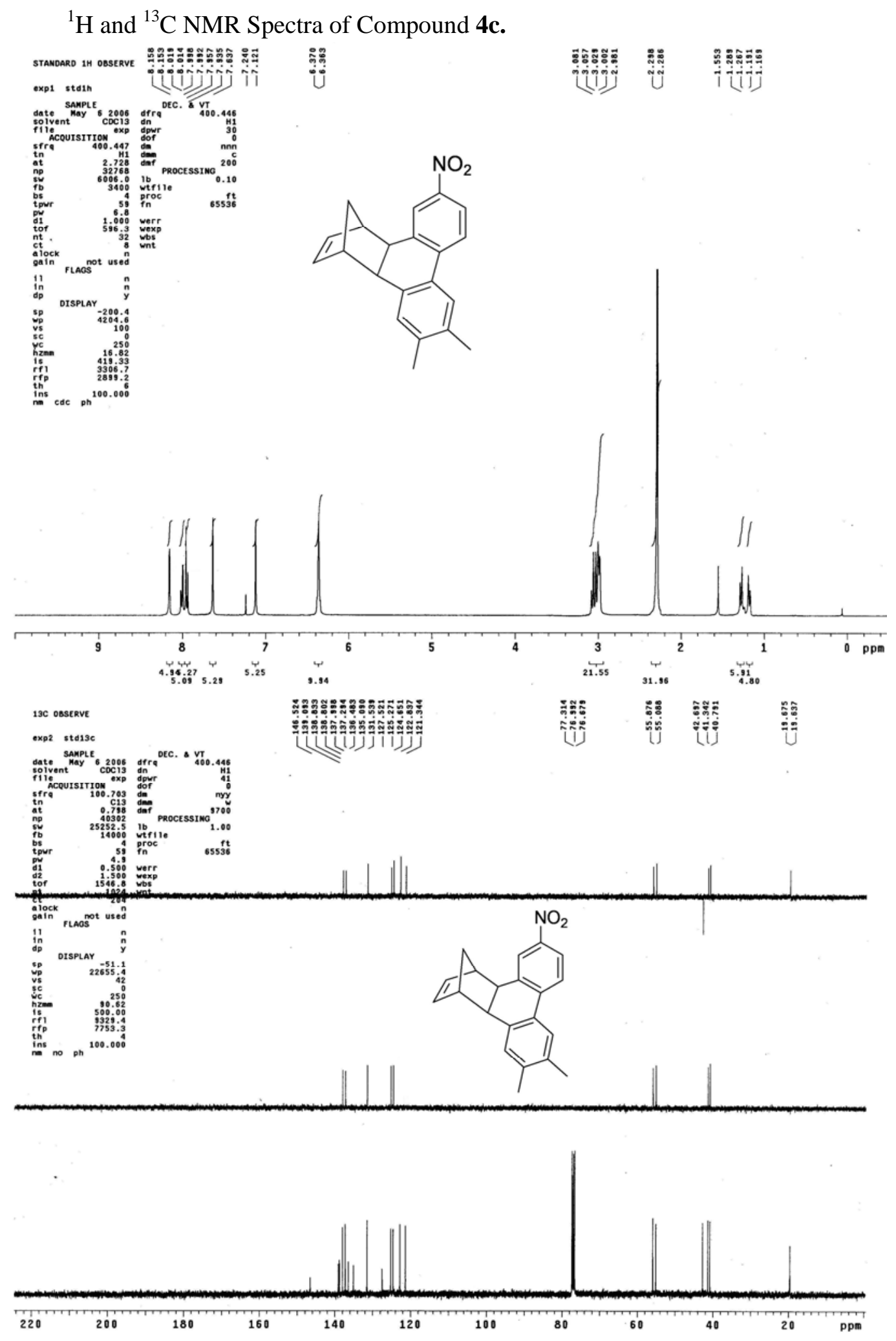
${ }^{1} \mathrm{H}$ and ${ }^{13} \mathrm{C}$ NMR Spectra of Compound 4d.
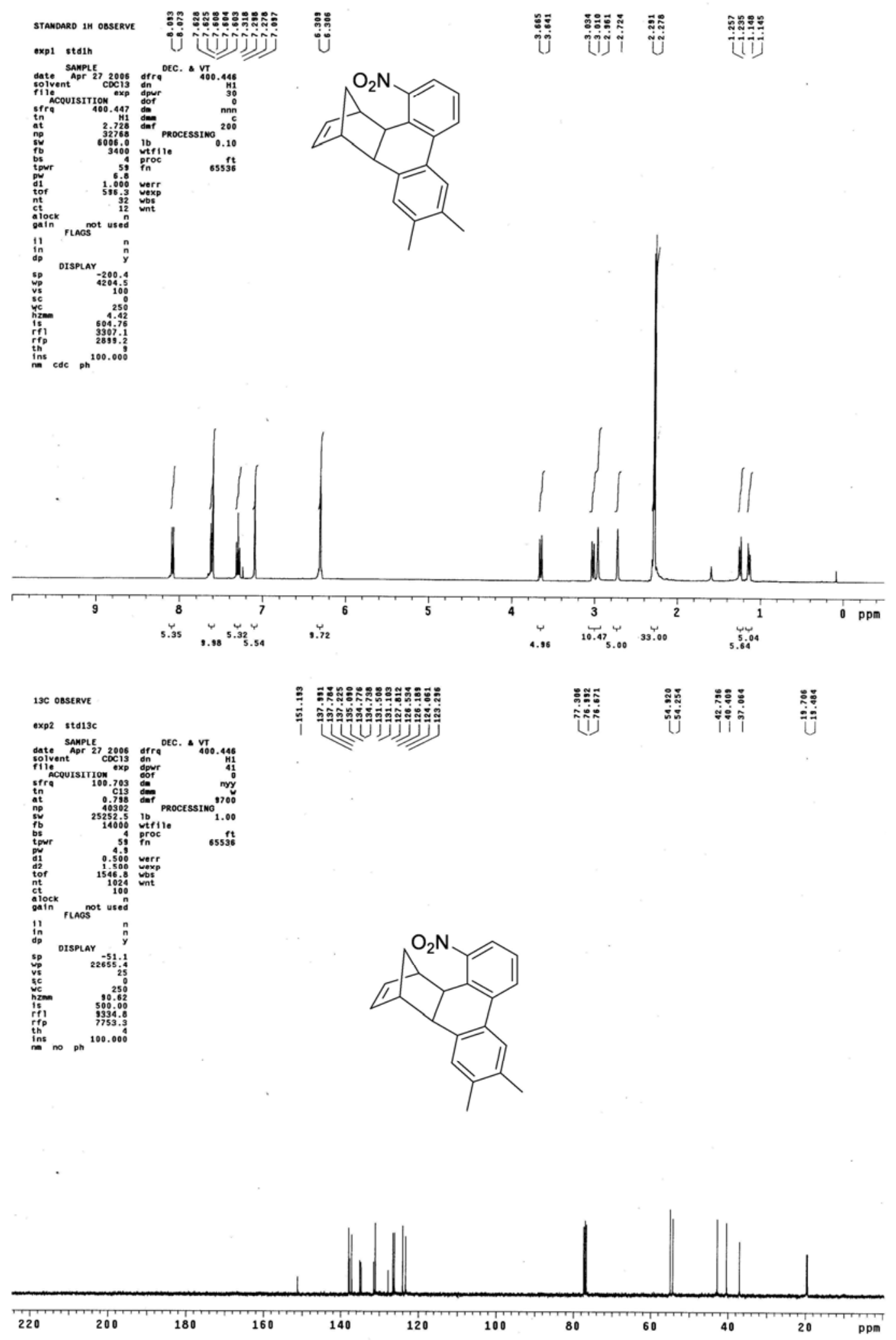
${ }^{1} \mathrm{H}$ and ${ }^{13} \mathrm{C}$ NMR Spectra of Compound $4 \mathbf{e}$.
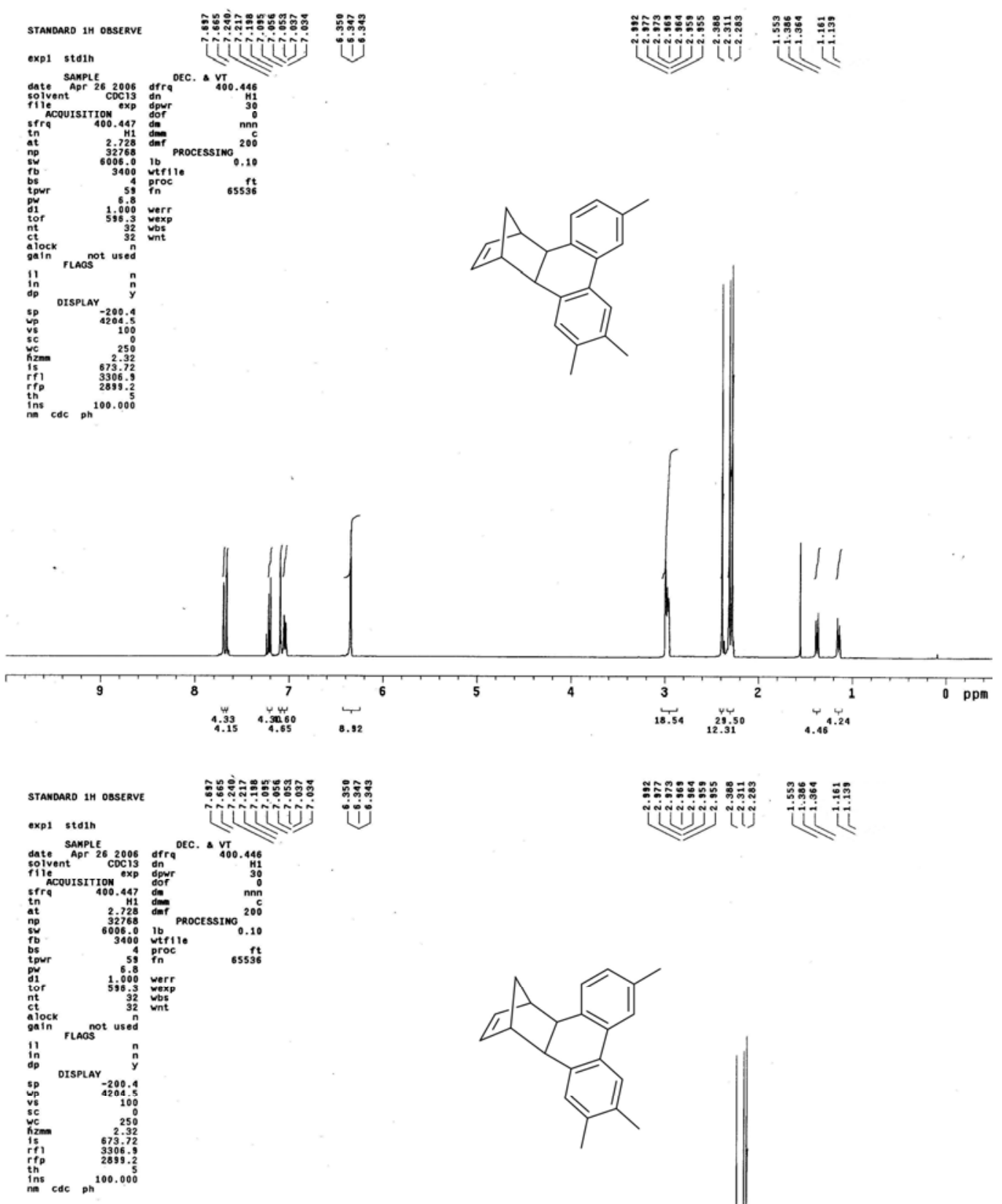
${ }^{1} \mathrm{H}$ and ${ }^{13} \mathrm{C}$ NMR Spectra of Compound $4 \mathbf{f}$.
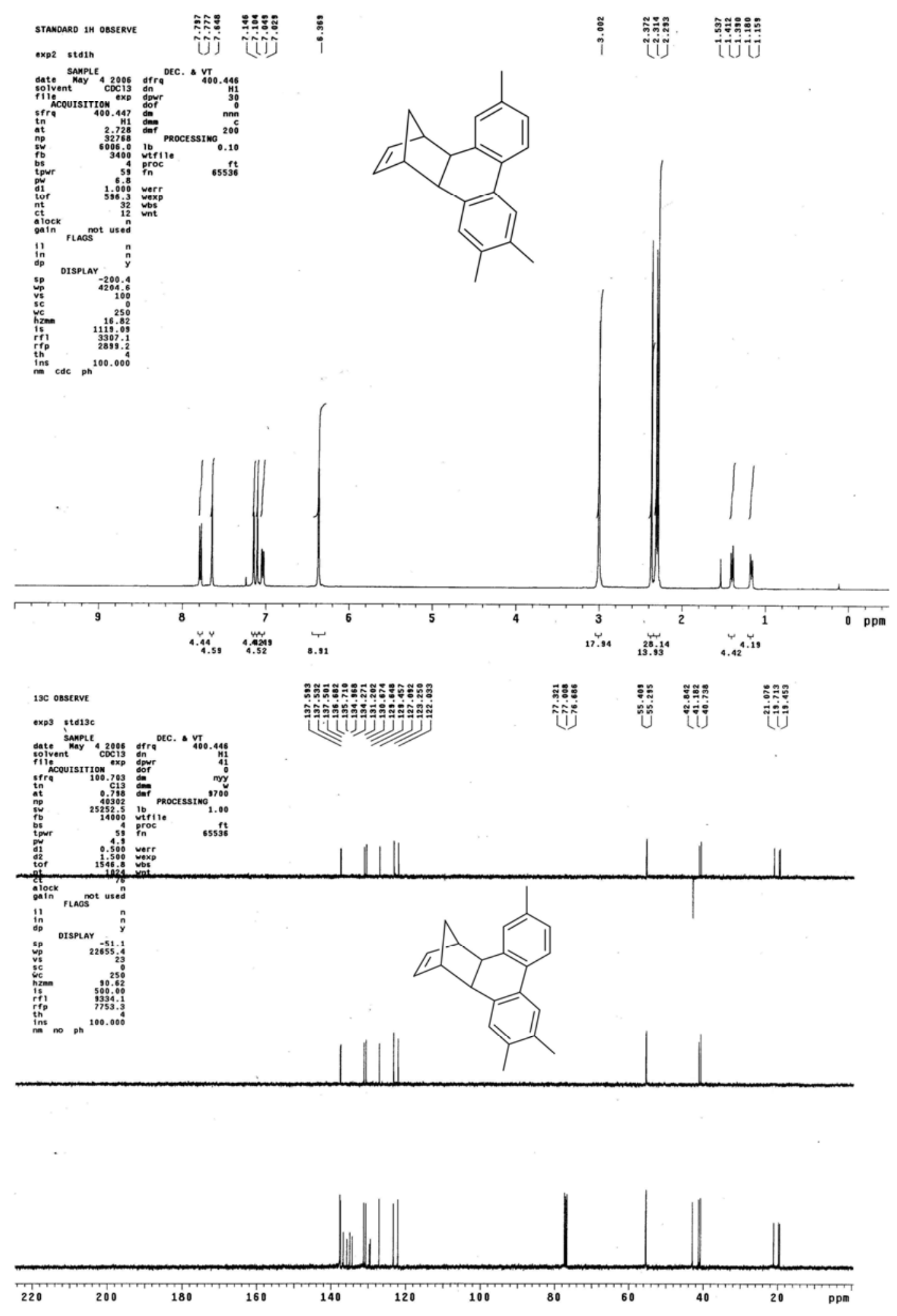
${ }^{1} \mathrm{H}$ and ${ }^{13} \mathrm{C}$ NMR Spectra of Compound $\mathbf{4 g}$.
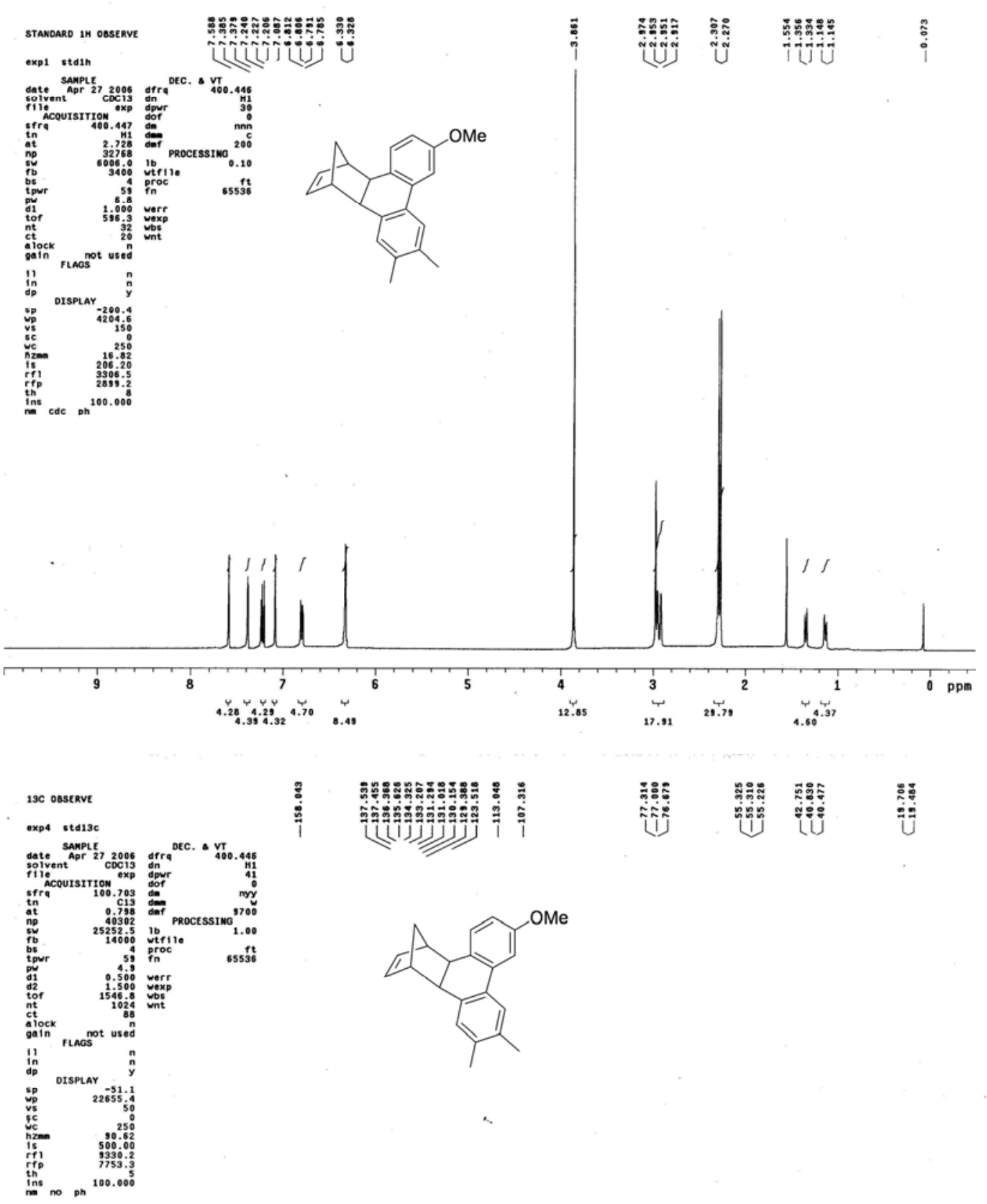

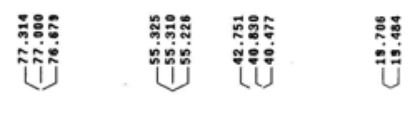

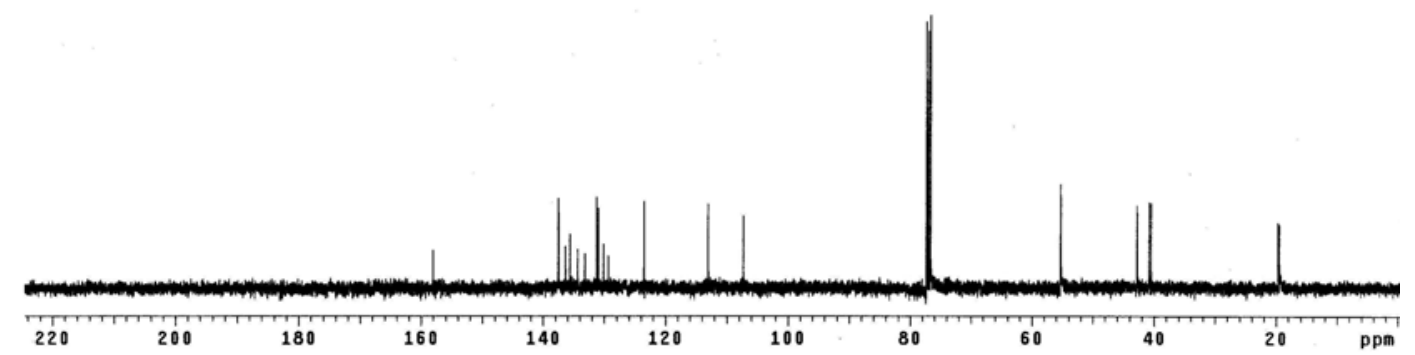


${ }^{1} \mathrm{H}$ and ${ }^{13} \mathrm{C}$ NMR Spectra of Compound $\mathbf{4 h}$.
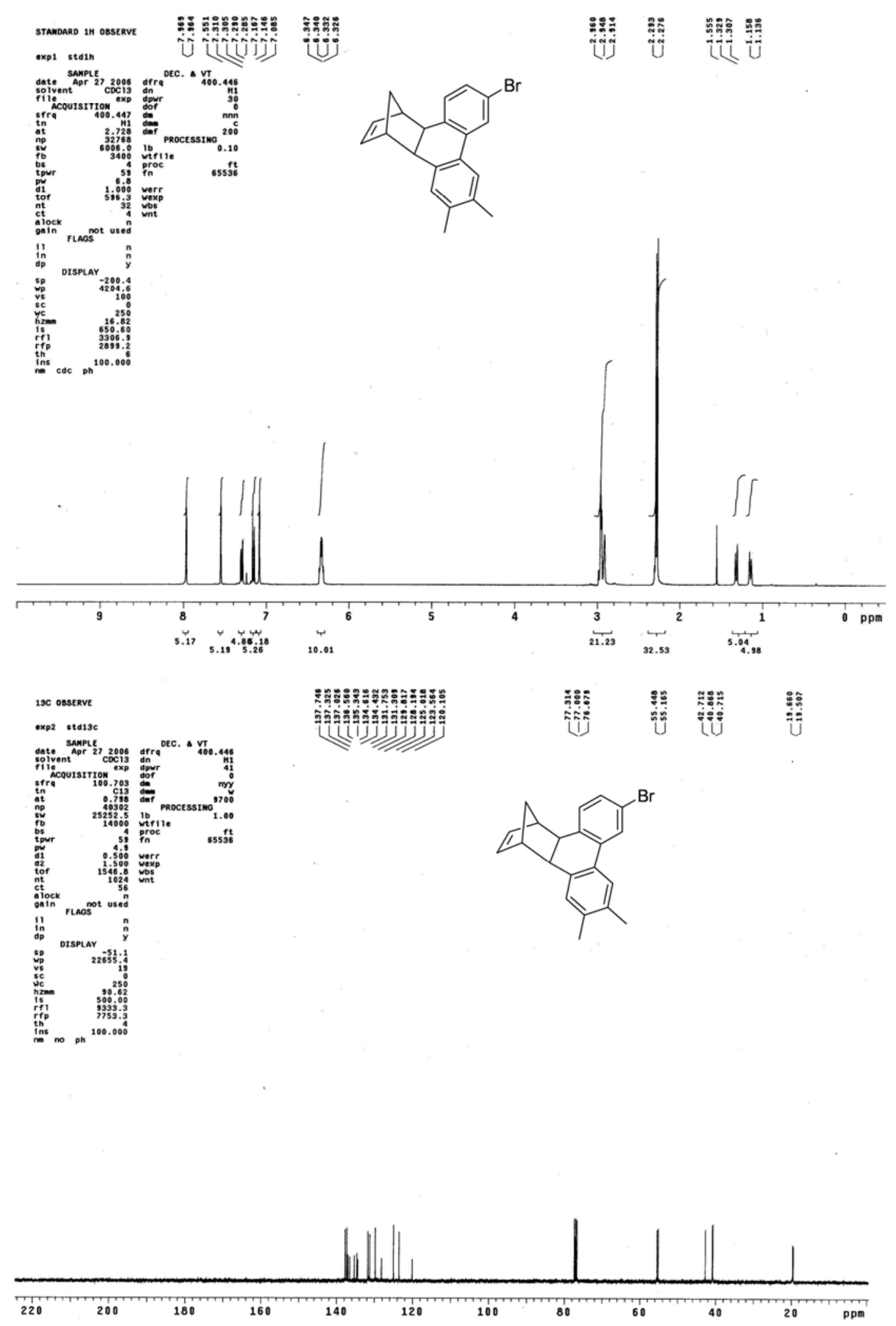
${ }^{1} \mathrm{H}$ and ${ }^{13} \mathrm{C}$ NMR Spectra of Compound $4 \mathbf{i}$.
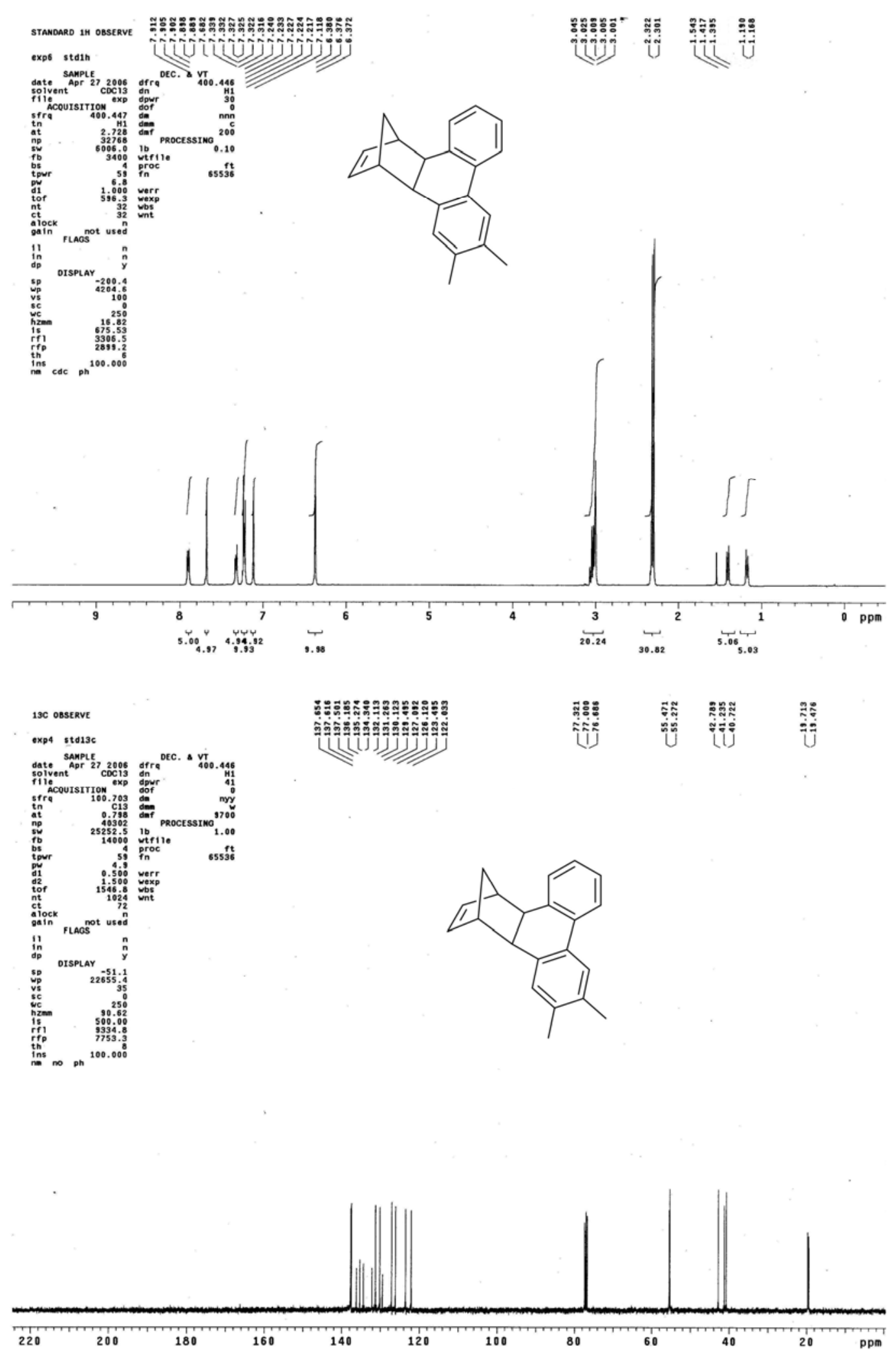
${ }^{1} \mathrm{H}$ and ${ }^{13} \mathrm{C}$ NMR Spectra of Compound $\mathbf{4 j}$.
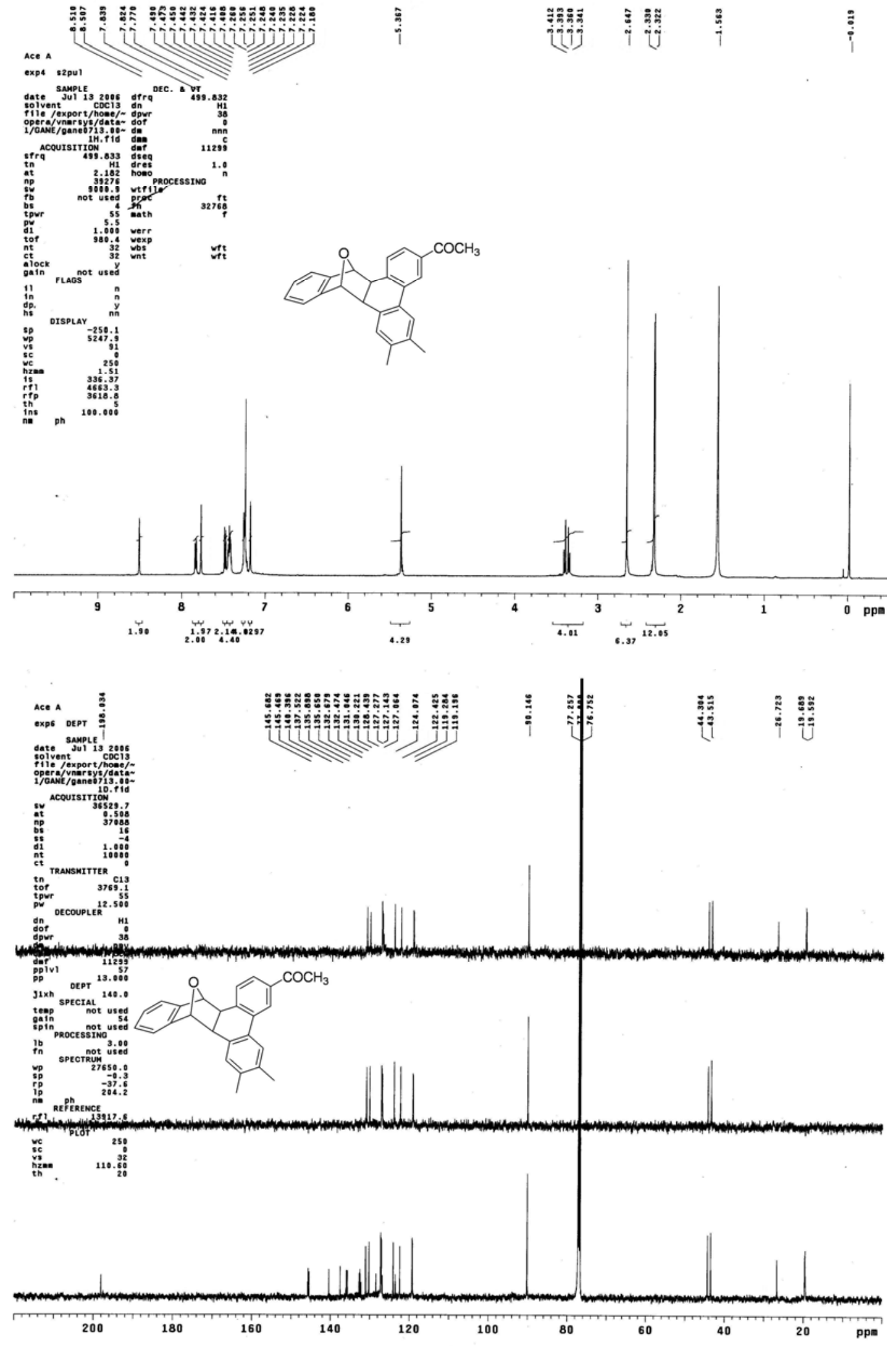
${ }^{1} \mathrm{H}$ and ${ }^{13} \mathrm{C}$ NMR Spectra of Compound $\mathbf{4 k}$.
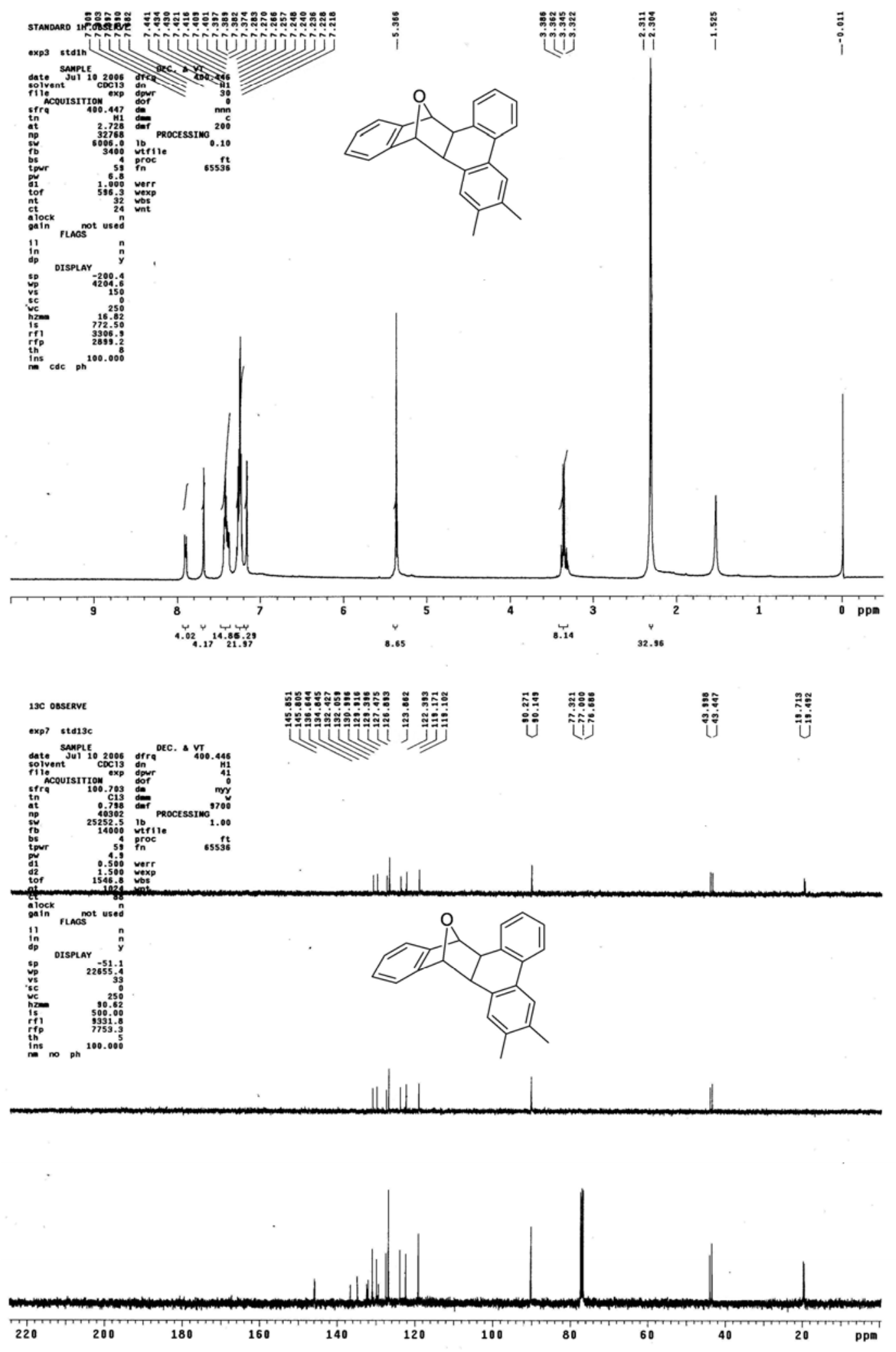
${ }^{1} \mathrm{H}$ and ${ }^{13} \mathrm{C}$ NMR Spectra of Compound $4 \mathbf{l}$.
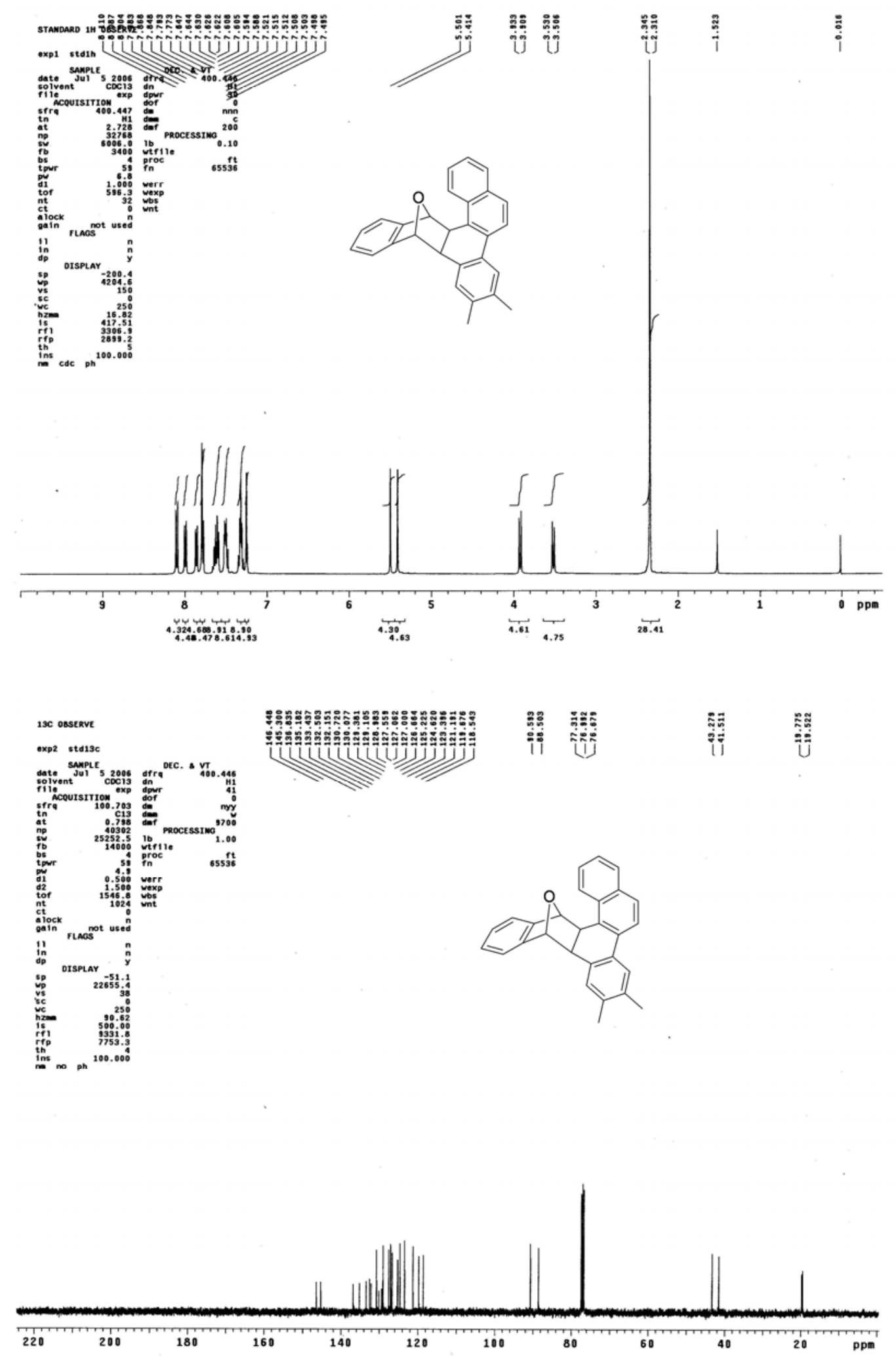
${ }^{1} \mathrm{H}$ and ${ }^{13} \mathrm{C}$ NMR Spectra of Compound $\mathbf{4 m}$.

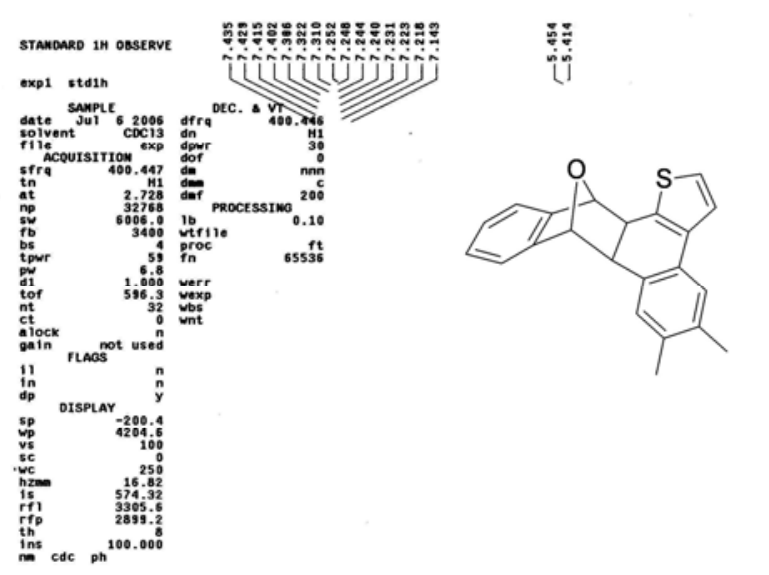

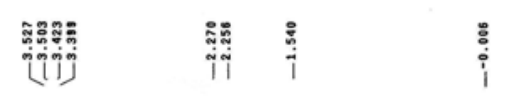
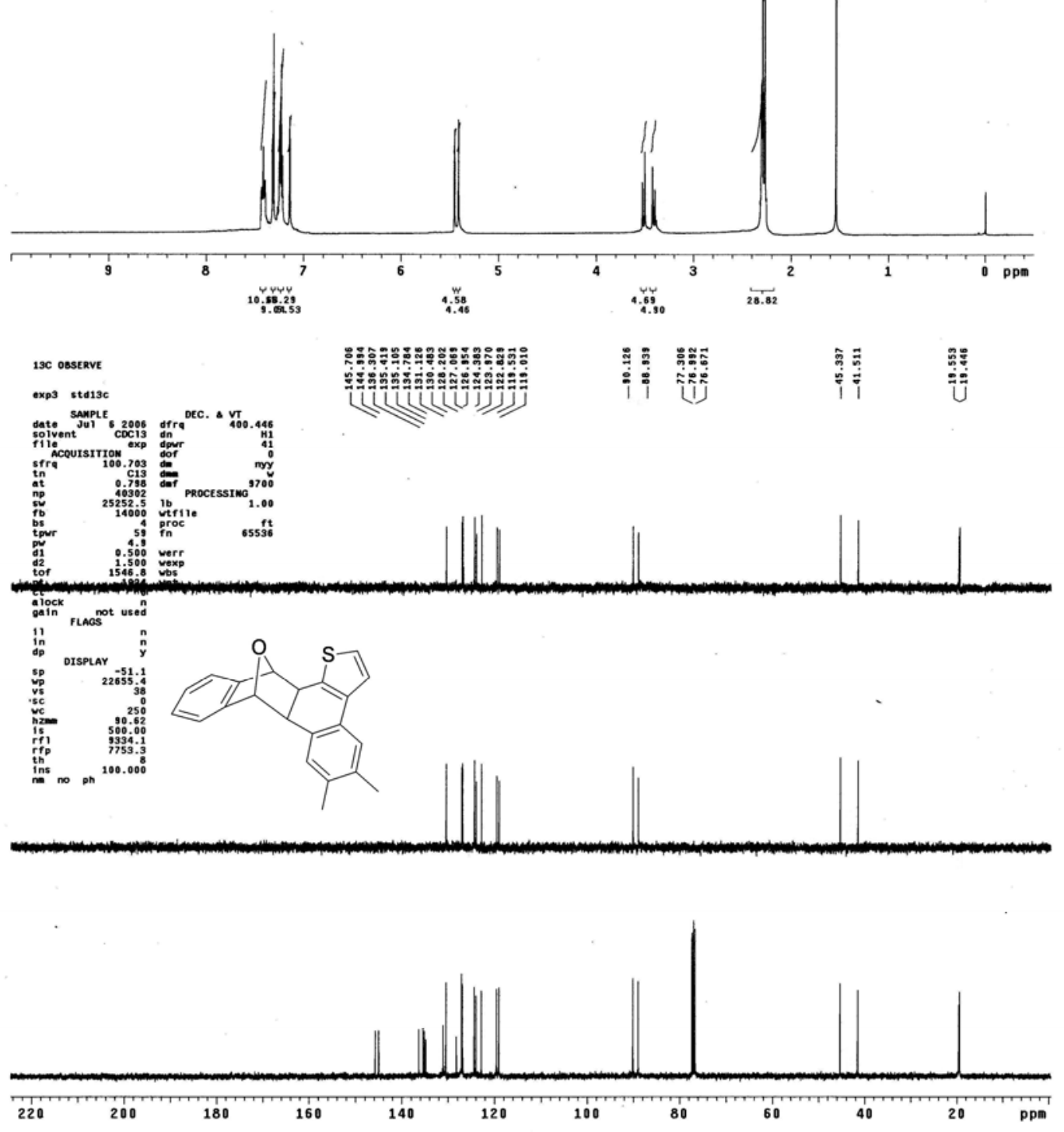

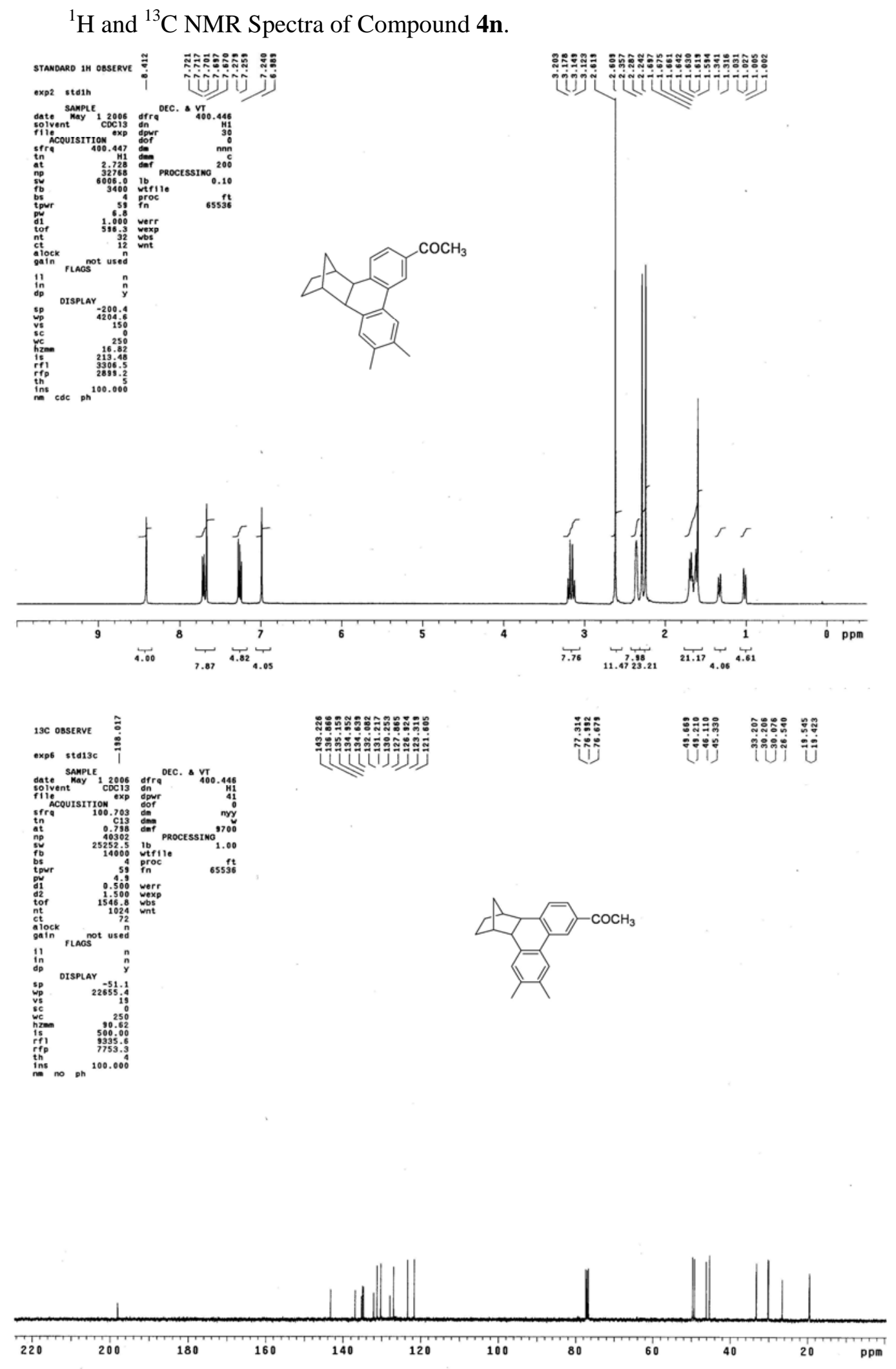
${ }^{1} \mathrm{H}$ and ${ }^{13} \mathrm{C}$ NMR Spectra of Compound $4 \mathbf{0 .}$
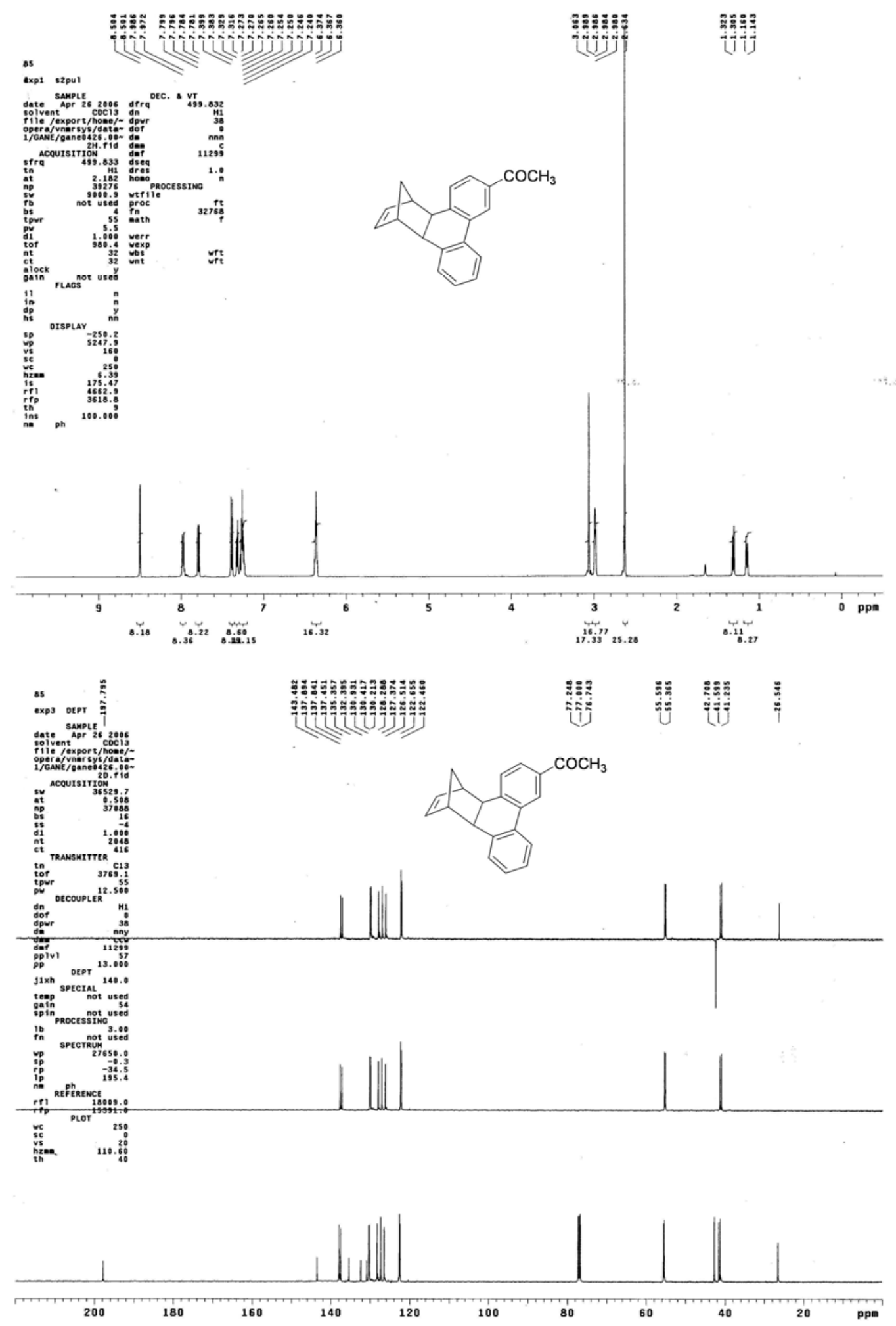
${ }^{1} \mathrm{H}$ and ${ }^{13} \mathrm{C}$ NMR Spectra of Compound $\mathbf{4 p .}$
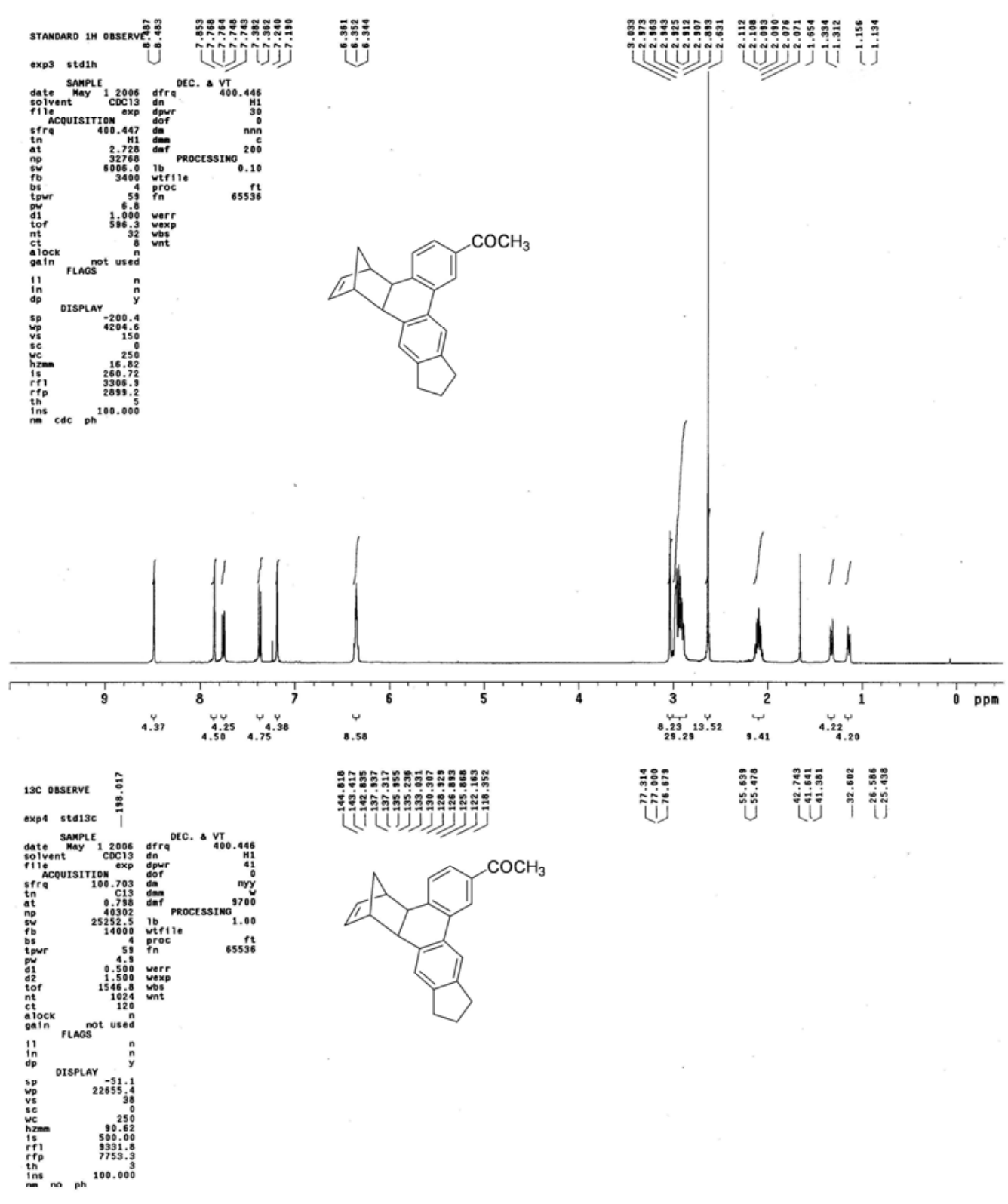

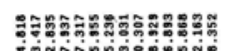

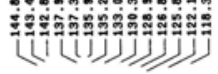

วัด

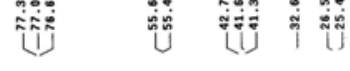
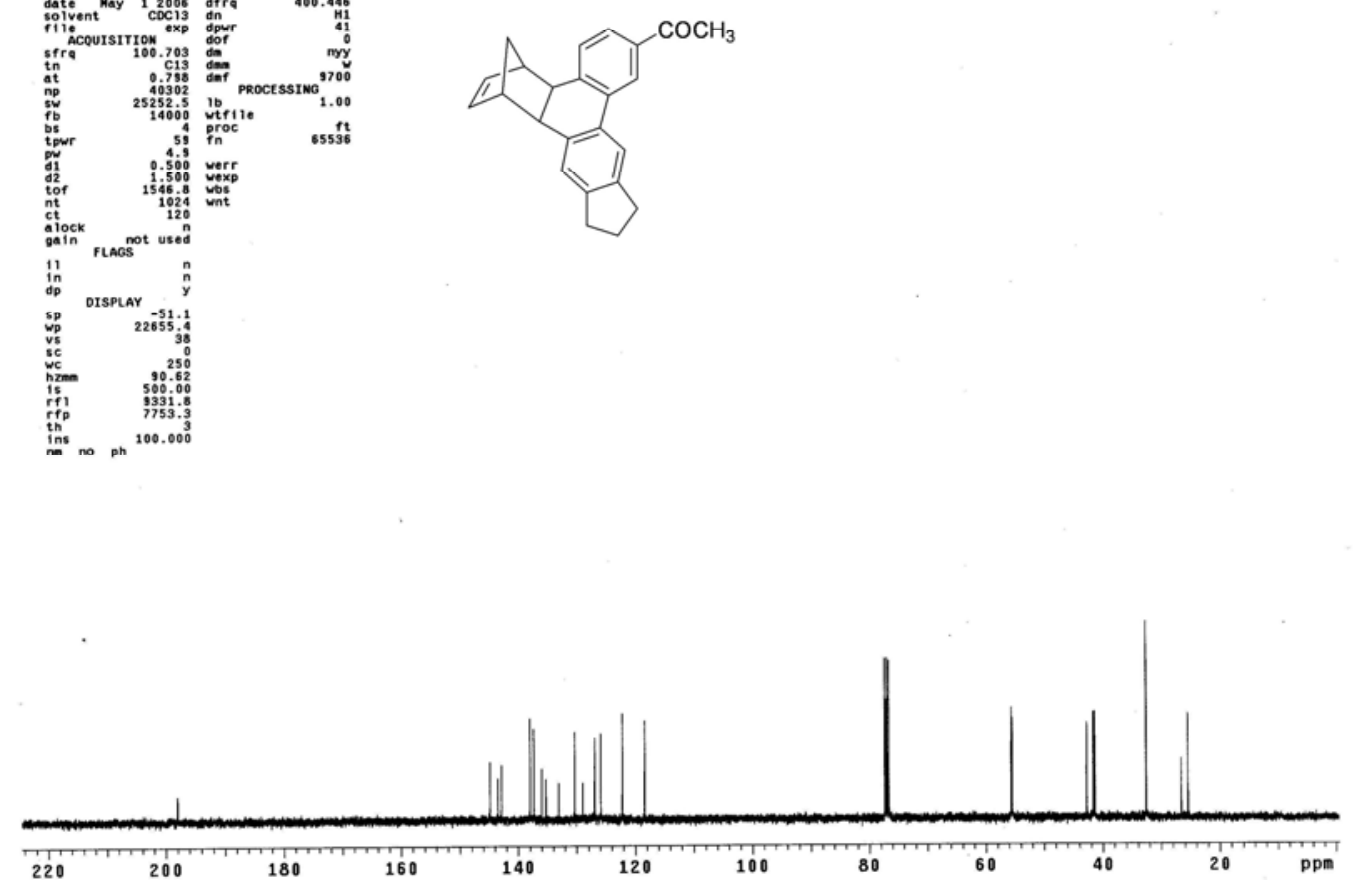


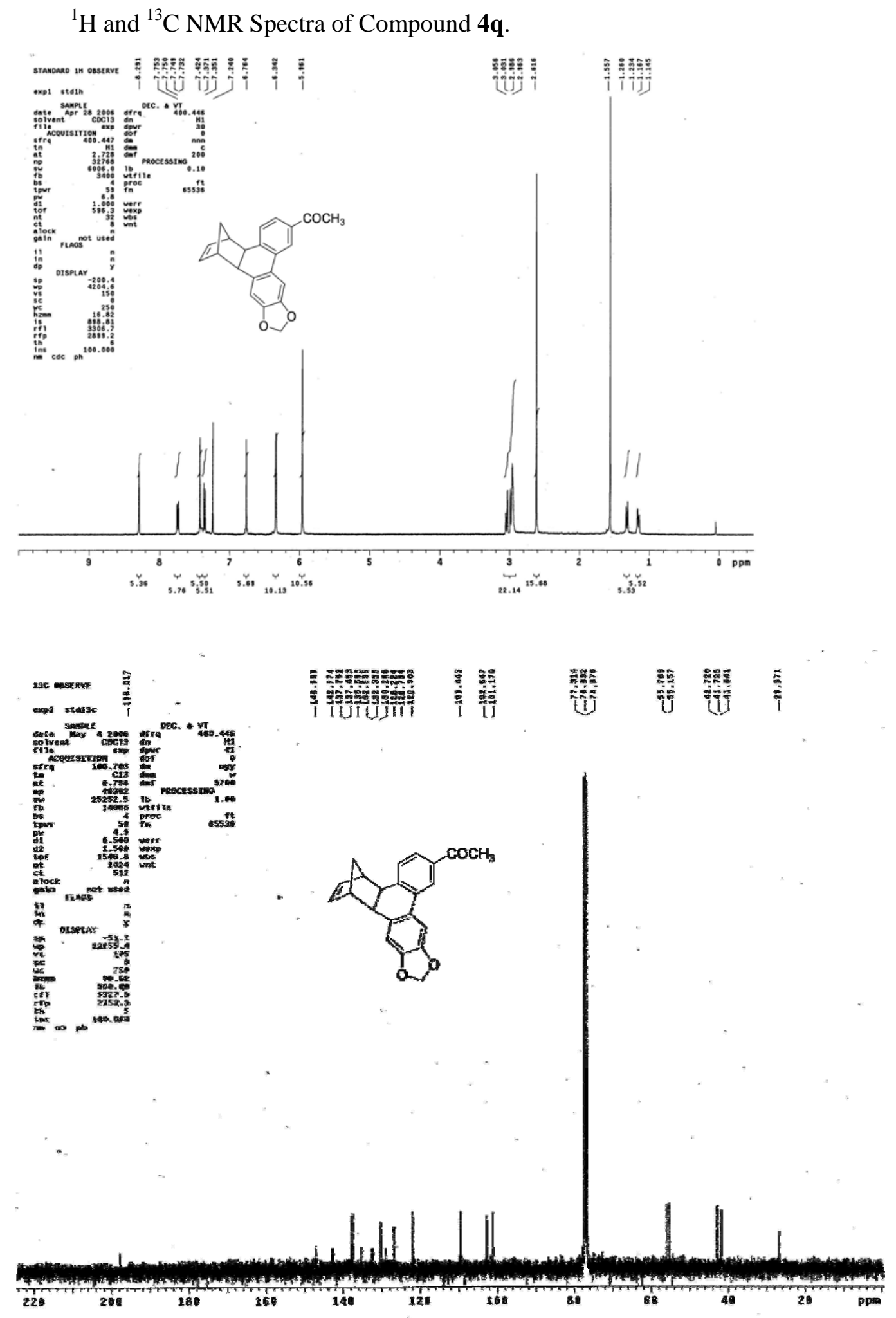



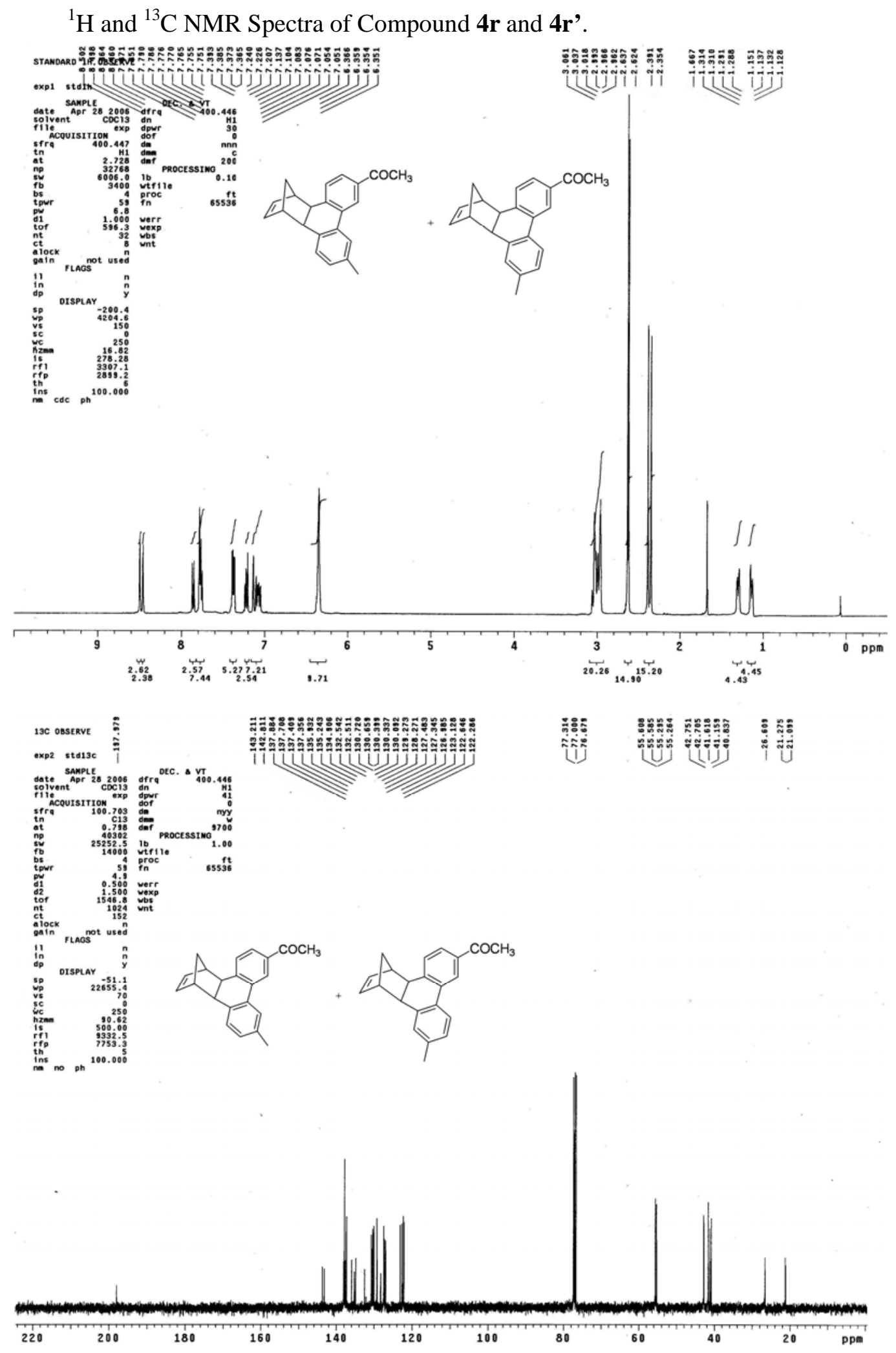
${ }^{1} \mathrm{H}$ and ${ }^{13} \mathrm{C}$ NMR Spectra of Compound 7a.
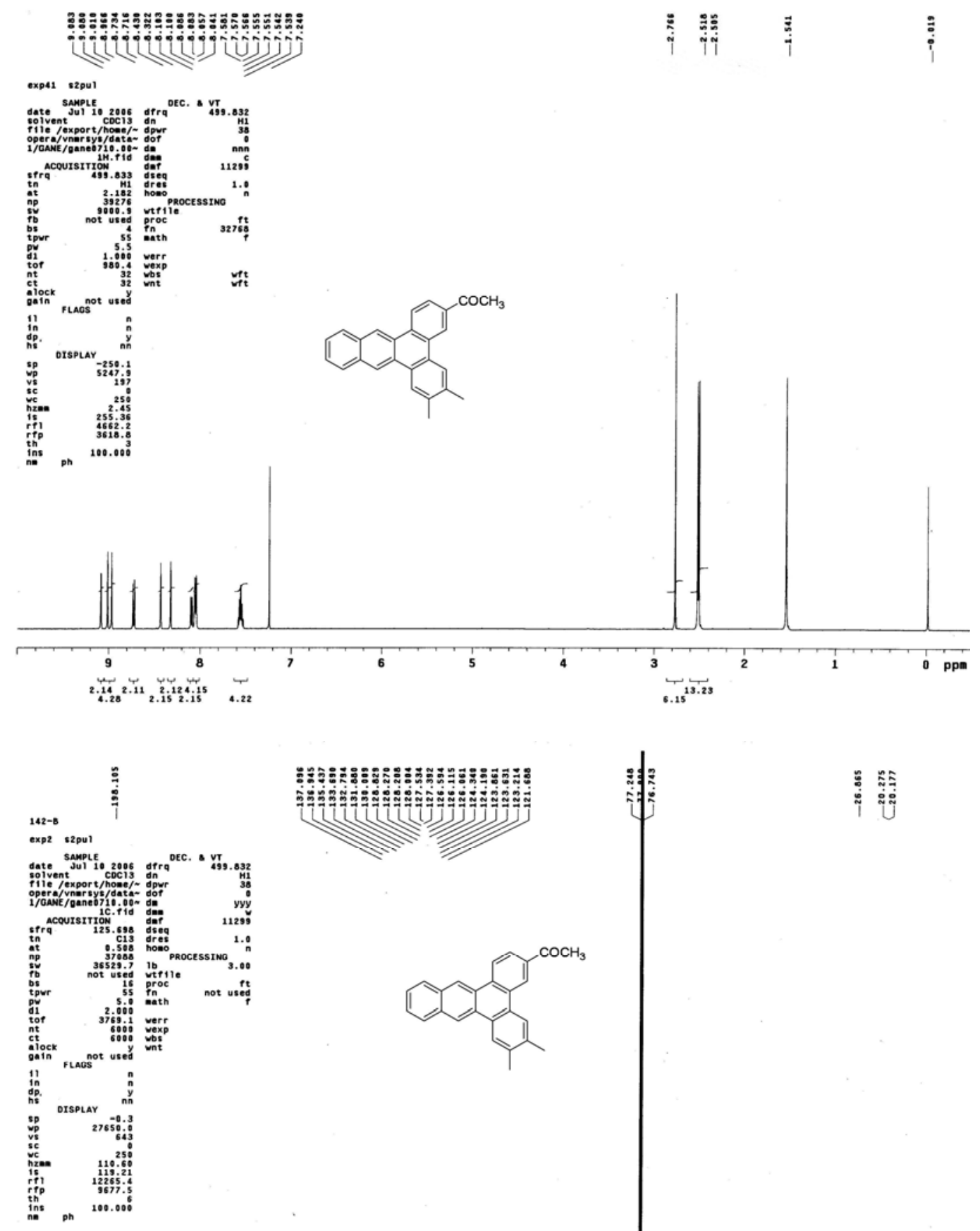

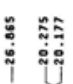

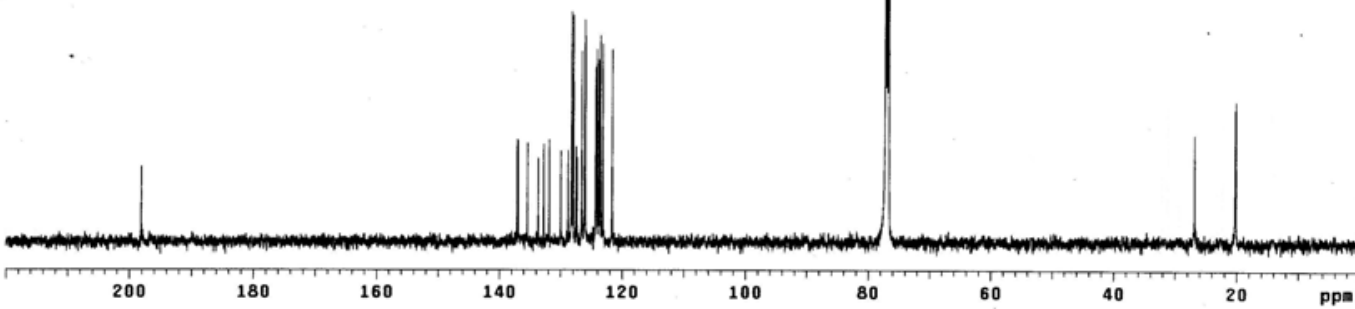


${ }^{1} \mathrm{H}$ and ${ }^{13} \mathrm{C}$ NMR Spectra of Compound $7 \mathbf{b}$.
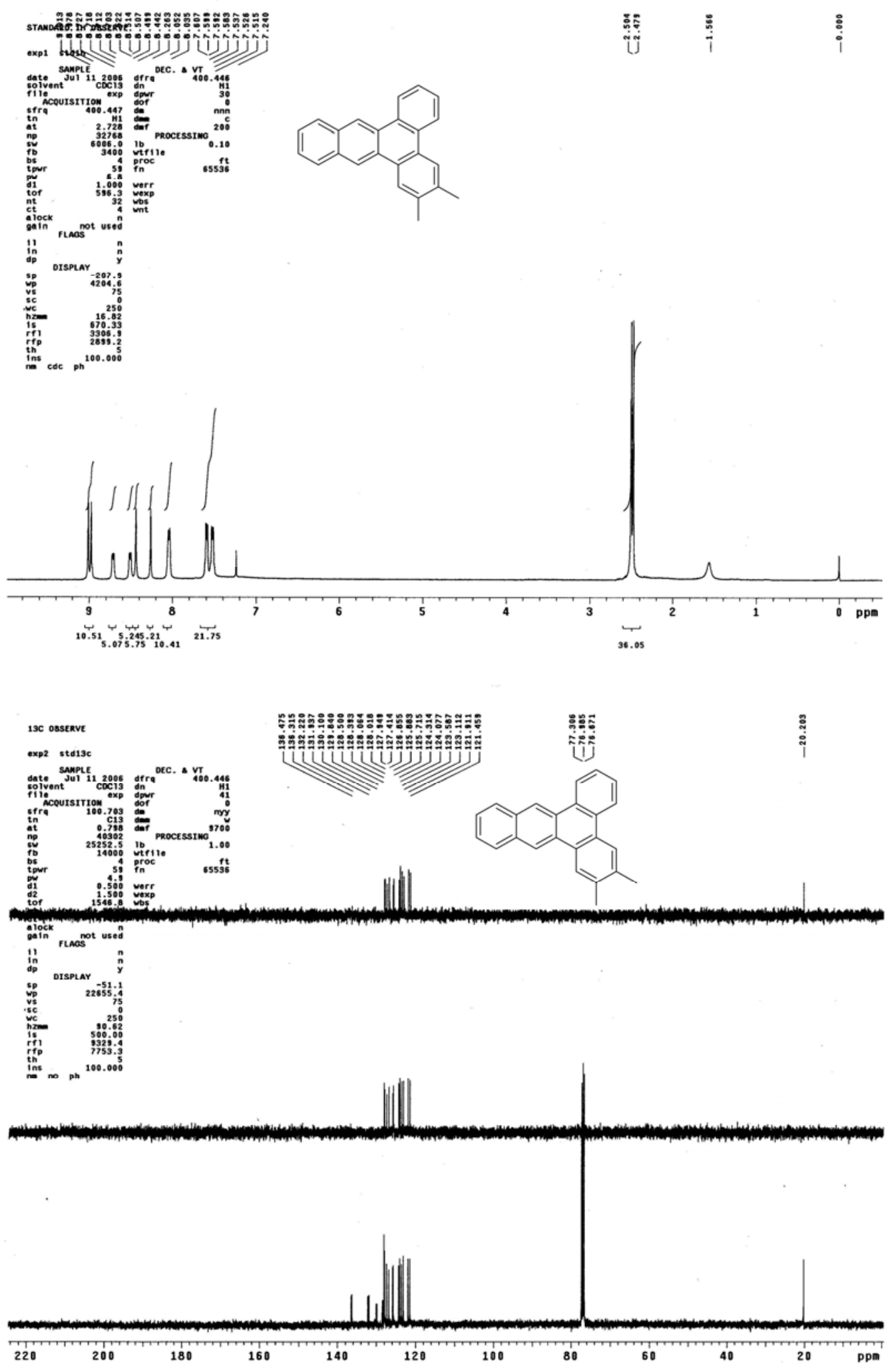